\title{
Cytosolic aggregates in presence of nontranslocated proteins perturb endoplasmic reticulum structure and dynamics
}

Debdatto Mookherjee ${ }^{1 \# *}$, Priyanka Majumder ${ }^{1,3 \#}$, Rukmini Mukherjee ${ }^{1,4 \#}$, Debmita Chatterjee $^{1 \$}$, Zenia Kaul ${ }^{1,5 \$}$, Subhrangshu Das ${ }^{2}$, Rachid Sougrat ${ }^{6}$, Saikat Chakrabarti ${ }^{2}$ and Oishee Chakrabarti ${ }^{1,7^{*}}$

${ }^{1}$ Biophysics \& Structural Genomics Division, Saha Institute of Nuclear Physics, 1/AF Bidhannagar, Kolkata - 700064, India.

${ }^{2}$ Structural Biology and Bioinformatics Division, CSIR-Indian Institute of Chemical Biology, CN 6, Sector V, Salt Lake, Kolkata - 700091, India.

${ }^{3}$ Department of Life Sciences, School of Natural Sciences, Shiv Nadar University, Dadri, UP, India.

${ }^{4}$ Buchmann Institute for Molecular Life Sciences, Frankfurt Am Main, Germany.

${ }^{5}$ Department of Microbiology, Immunology, and Cancer Biology, University of Virginia School of Medicine, USA.

${ }^{6}$ Imaging and Characterization Lab, 4700 King Abdullah University of Science and Technology, Thuwal 23955-6900, Kingdom of Saudi Arabia.

${ }^{7}$ Homi Bhabha National Institute.

\#These authors contributed equally to this work

${ }^{\$}$ These authors contributed equally to this work

*Corresponding authors:

Oishee Chakrabarti

oishee.chakrabarti@saha.ac.in

Debdatto Mookherjee

debdatto.mookherjee@saha.ac.in

Running Title: Nontranslocated proteins perturb ER

Key words: Nontranslocated protein precursor, cytosolic aggregates, endoplasmic reticulum, remodelling.

This article has been accepted for publication and undergone full peer review but has not been through the copyediting, typesetting, pagination and proofreading process which may lead to differences between this version and the Version of Record. Please cite this article as doi: $10.1111 /$ tra. 12694 
Abbreviations: AD: Alzheimer's disease, ALS: amyotrophic lateral sclerosis, ATP: Adenosine triphosphate, ATRA: all-trans retinoic acid, CBP: CREB binding protein, CFP: cyan fluorescent protein, CRFR1: corticotrophin-releasing factor receptor1, EndoH: Endoglycosidase H, ER: endoplasmic reticulum, ERAD: Endoplasmic-reticulum-associated protein degradation, FRAP: fluorescent recovery after photobleaching, FTD: Fronto-temporal dementia, GFP: green fluorescent protein, HD: Huntington's disease, HEPES: 4-(2hydroxyethyl)-1-piperazineethanesulfonic acid, Htt: huntingtin, IEM: immuno electron microscopy, Ifn $\gamma$ : interferon gamma, LNPK: Lunapark, Opn: osteopontin, PD: Parkinson's disease, Prl: prolactin, PrP: prion protein, pQC: preemptive quality control, RAPP: Regulation of Aberrant Protein Production, RFP: red fluorescent protein, RHD: C-terminal reticulon homology domain, ROI: region of interest, ROS: reactive oxygen species, RTN: reticulon, SDS-PAGE: sodium dodecyl sulfate polyacrylamide gel electrophoresis, SRP: signal recognition particle, UPS: ubiquitin-proteasome system, VSVG: vesicular stomatitis virus $\mathrm{G}$ protein tagged, wt: wild type.

\title{
Synopsis
}

Cytosolic protein aggregates and endomembrane damage are hallmarks of neurodegenerative diseases. Here we show that in presence of intracellular aggregates when protein translocation efficiencies are compromised, the ER structure and dynamics are affected. Under such circumstances, the ER gets vacuolated, relative distribution of RER and SER is altered, and three way junctions of tubular ER are destabilised. This highlights a lesser known efect of cytosolic aggregates on nontranslocated precursor proteins - how their presence and persistence affects endomembrane dynamics.

\section{Graphical Abstract}

\begin{abstract}
Presence of cytosolic protein aggregates and membrane damage are two common attributes of neurodegenerative diseases. These aggregates delay degradation of nontranslocated protein precursors leading to their persistence and accumulation in the cytosol. Here we find that
\end{abstract}


cells with intracellular protein aggregates (of cytosolic prion protein or huntingtin) destabilise the endoplasmic reticulum (ER) morphology and dynamics when nontranslocated protein load is high. This affects trafficking of proteins out from the ER, relative distribution of the rough and smooth ER and three-way junctions that are essential for the structural integrity of the membrane network. The changes in ER membranes may be due to high aggregation tendency of the ER structural proteins - Reticulons, and altered distribution of those associated with the three-way ER junctions - Lunapark. Reticulon4 is seen to be enriched in the aggregate fractions in presence of nontranslocated protein precursors. This could be mitigated by improving signal sequence efficiencies of the proteins targeted to the ER. These were observed using PrP variants and the seven-pass transmembrane protein (CRFR1) with different signal sequences that led to diverse translocation efficiencies. This identifies a previously unappreciated consequence of cytosolic aggregates on nontranslocated precursor proteins - their persistent presence affects ER morphology and dynamics. This may be one of the ways in which cytosolic aggregates can affect endomembranes during neurodegenerative disease.

\section{Introduction}

Cytosolic protein aggregates have several detrimental effects on cellular physiology. Neurodegenerative diseases like Huntington's disease (HD), Alzheimer's disease (AD), Parkinson's disease (PD), Fronto-temporal dementia (FTD) have protein aggregation as their chief pathophysiology. ${ }^{1}$ Neurofibrillary tangles of Tau, Huntington deposits, $\alpha$-synuclein containing Lewy bodies alter fundamental cellular processes like protein quality control, intracellular transport of cargo, mitochondrial ATP generation and lysosomal function. ${ }^{2-6}$ Aggregates can be juxtanuclear or perivacuolar. This often depends on their aggregation state. Soluble aggregates concentrate in the juxtanuclear region where they can be removed by the proteasomal machinery, while terminal aggregates like huntingtin protein aggregates are targeted to perivacuolar compartments. ${ }^{7}$ Huntingtin aggregates have been shown to block nucleopores affecting nucleocytoplasmic transport. ${ }^{8}$ 
Often, these effects are not solely determined by the aggregates themselves; the cellular milieu plays an important role in determining the degree of damage caused by the aggregate. Previous studies showed the role of nontranslocated proteins in growth and persistence of aggregates. ${ }^{9,10}$ Protein translocation involves recognition of signal sequences on ribosomebound nascent chains by the signal recognition particle (SRP). Efficiency of protein translocation depends on the strength of the signal sequence present at the $\mathrm{N}$ terminus of the protein. ${ }^{11,12}$ Some proteins like osteopontin (Opn) or prolactin (Prl) have strong hydrophobic signal sequences which cause efficient translocation of the protein into the endoplasmic reticulum (ER). ${ }^{13}$ Others like interferon gamma (Ifn $\left.\gamma\right)$ and prion protein (PrP), inspite of having hydrophobic signal sequences are translocated into the ER inefficiently. ${ }^{14}$ This could be due to inherent incompetence of their targeting and translocation ${ }^{13,15}$ or as a consequence of regulated translocation ${ }^{16}$ - whichever may be the cause, it results in a significant proportion of the protein along with the uncleaved hydrophobic polypeptide to remain in the cytosol. ${ }^{9}$ A substantial proportion of proteasomal substrates may consist of proteins that, like PrP, fail to translocate with high fidelity. These are subjected to a preemptive quality control (pQC) pathway and are degraded by the ubiquitin-proteasome system (UPS). Nontranslocated proteins are also degraded by translational quality control pathways like RAPP (Regulation of Aberrant Protein Production). ${ }^{17}$ In presence of cytosolic aggregates nontranslocated proteins which are not efficiently degraded have been shown to increase the growth and persistence of cytosolic aggregates. ${ }^{9}$ Using a wide range of PrP variants that differ in the strength of their signal sequences and hence protein translocation efficiencies, it has been shown that nontranslocated secretory and membrane proteins tend to stabilize and perpetuate in the presence of cytosolic pre-existing aggregates in cells. ${ }^{9}$ When the weak signal sequence of endogenous PrP was replaced with that of Prl (which is a strong signal sequence), the colocalisation in aggregates is significantly reduced. Instead, if the signal sequence of Ifn $\gamma$ be used, translocation efficiency is poor. ${ }^{14}$ A fusion PrP protein with Ifn $\gamma$ signal sequences (Ifn $\gamma$-PrP) mimics the pQC pathway by undergoing efficient SRP-dependent targeting to the translocon but inefficient translocon gating, and hence has poor access to the ER lumen, release into the cytosol and ubiquitination. ${ }^{13,14}$ This suggested that presence of Ifn $\gamma$ signal sequence (which is weaker than that of $\mathrm{PrP}$ ) would enhance the "co-aggreagation phenotype". Interestingly, co-association of nontranslocated secretory and membrane proteins in pre-existing cytosolic aggregates also lead to differences in the cellular morphology, particularly the architecture of ER membranes - the extent of these structural aberrations in cells being governed by the translocation efficiencies of the aforementioned proteins. ${ }^{9}$ 
The ER membrane is a continuous network of tubules, cisternae, matrices and sheets. The rough ER has a sheet like morphology and has an important role in protein translocation, folding and trafficking. The smooth ER consists of tubules and is important for lipid biosynthesis and calcium signalling. The reticulon (RTN) family of proteins regulate ER shape by controlling membrane curvature via scaffolding and hydrophobic wedging. ${ }^{18}$ In mammals, this consists of 4 proteins RTN1-4, which exist as several isoforms generated by alternate splicing. They have a C-terminal reticulon homology domain (RHD), consisting of two hydrophobic regions flanking a hydrophilic loop. The RHD hydrophobic regions are longer than usual transmembrane domains spanning 30-35 amino acids. ${ }^{19,20}$ These longer transmembrane domains may be important to their function. The topology of reticulons in membranes is disputed but recent reports suggest that both the amino and carboxy termini are on the cytosolic side of the ER membrane..$^{20}$ RTN mediated membrane remodelling is a relatively new area of research and how the ER changes shape in response to cellular cues is mostly unknown. ${ }^{21,22}$ This highly dynamic reticulum is constantly remodelled to carry out various functions. Reports on membrane remodelling in presence of cytosolic aggregates are few and how aggregates modify membrane architecture remains an open question.

ER dysfunction in terms of protein trafficking defects are common in most neurodegenerative diseases but the change in ER structure has not been studied in detail. For example, RTN3 levels change in AD but the role of RTNs in AD remains obscure. ${ }^{23}$ Its presence has also been noted in Lewy bodies in brain sections of PD patients, in mouse models of amyotrophic lateral sclerosis (ALS) and also regulates autophagy and clearance of cytosolic prion aggregates. ${ }^{24-26}$ While the assembly of aggregates, their distribution and degradation are widely studied, their effect on ER membrane architecture and dynamics remain ambiguous.

Here we show the effect of nontranslocated proteins and cytosolic aggregates on the ER architecture in the cell. When cells with pre-existing aggregates are transfected with $\operatorname{PrP}$ variants having a weak signal sequence (heterologous $\operatorname{PrP}$, and Ifn $\gamma$-PrP), the ER membrane loses its distinct reticular morphology, undergoing vacuolation and remodelling which changes its proportion between sheets and tubules. This effect is mitigated when the PrP signal sequence is replaced with a stronger Prolactin (Prl) signal sequence. The functional consequence of this ER remodelling is its effect on protein trafficking from the ER to Golgi as well as its effect on the stability of the three way junctions of tubular ER. RTN4 was noted to be present in cytosolic PrP aggregates in cells with high load of nontranslocated proteins 
than in control cells. This suggests that changes in ER morphology is due to sequestration of ER structural proteins in aggregates in PrP and Ifn $\gamma$-PrP expressing cells which is minimal in aggregate bearing cells that express Prl-PrP. This study supports the hypothesis that nontranslocated proteins lead to growth and persistence of cytosolic aggregates and this eventually affects ER morphology and dynamics.

\section{Results}

\section{Experimental logic}

This study utilises a wide range of PrP variants and other constructs that have been extensively characterised in earlier studies. ${ }^{9,14,16,27}$ The three types of PrP that have been used in this study are Prl-PrP (having the signal sequence of bovine prolactin which is a strong signal sequence for protein translocation), wt-PrP (having the endogenous PrP signal sequence-which is a weaker signal sequence) and $\operatorname{Ifn} \gamma-\operatorname{PrP}$ (having the sequence of interferon gamma which is weaker than both the Prl and PrP signal sequences). ${ }^{13}$ Briefly, these variants differ in their localization and biochemical properties. Due to inefficiencies in translocation of proteins trafficking through the secretory pathway, a fraction remains nontranslocated before its triage for degradation. This can get recruited into preexisting aggregates or have soluble residence in the cytosol. Biochemical analyses of the steady state levels shows presence of increased levels of unglycosylated and hence nontranslocated forms of PrP in the presence of constructs with inefficient signal sequences (Supplementary Figure1A) - here though the total levels of Ifn $\gamma$-PrP are lower than the two other PrP variants, it generated the highest level of non-glycosylated and therefore presumably non-translocated, precursor. 


\section{ER remodelling in cells with nontranslocated $P r P$ and cyPrP aggregates}

We began our study by analysing the effect of nontranslocated protein accumulation on the ER morphology. For this we used the laboratory generated construct of monomeric fluorescent protein (mFP)-tagged wild-type $\mathrm{PrP}$ with residues 40-231 ( $\mathrm{PrP}_{40-231-\mathrm{mFP}}$ ) or mFP-tagged $\mathrm{Htt}_{\mathrm{Q} 103}$, both of which have been shown to form intracellular aggregates. ${ }^{9}$ The aggregation-prone artificial PrP construct is henceforth referred to as cyPrP. It has been previously reported that in a fraction of cells expressing PrP and cyPrP aggregates, the nontranslocated PrP colocalised with the aggregates and stabilised them. There were also cells with little or no evidence of coaggregation detected. In all these cases, in presence of cytosolic aggregates, it was observed that initially, the expressed protein had a nonnuclear reticular localisation consistent with the ER. However, over a period of time, a substantial proportion of this was distributed diffusely in the nucleocytoplasmic compartment in addition to its expected ER localization. ${ }^{9}$ (Supplementary Figures 1B and C) This was irrespective of the choice of proteins that formed the initial aggregates. Like before, we utilised the native protein, corticotropin-releasing factor receptor type 1 (CRFR1), a G protein-coupled receptor which has an inefficient signal sequence ${ }^{9,16}$ to test this hypothesis. Again, presence of the native CRFR1 signal sequence led to nucleocytoplasmic protein distribution over time; while swapping with the Prl signal sequence (thereby generating Prl-CRFR1), alleviated this effect. This raised the possibility that prolonged cytosolic retention of membrane targeted proteins could eventually disrupt some of the endomembrane architecture.

To verify this, HeLa cells were cotransfected with ER-GFP ${ }^{28}$, RFP tagged cyPrP and 3 variants of PrP differing in their signal sequences. Control cells expressed RFP tagged cyPrP only. The ER morphology in these cells was observed using confocal imaging $48 \mathrm{~h}$ after transfection. The ER in Prl-PrP expressing cells appeared to be a connected branched network which is similar to cells expressing cyPrP only. Upon transfection of wt-PrP, the ER morphology was fragmented with formation of vacuoles in $\sim 50 \%$ cells. Ifn $\gamma$-PrP transfection led to loss of the reticular nature of the ER in $\sim 70 \%$ cells; these had highly fragmented ER with a lot of vacuolar spaces (Figure 1A). Live cell imaging of cells cotransfected with cyPrP, PrP variants and ER-GFP showed that ER dynamics (as quantitated by successful fusion events) was slower in cells expressing wt-PrP and Ifn $\gamma$-PrP as compared to those expressing Prl-PrP (Figure 1B). This difference in ER dynamics was also reflected in a FRAP (fluorescent recovery after photobleaching) assay. FRAP recovery rates of ER-GFP in Prl- 
PrP, wt-PrP, and Ifn $\gamma$-PrP were different when similarly sized ROIs were photobleached and tracked over time (Figure 1C). While recovery is $~ 80 \%$ in Prl-PrP expressing cells, it was only $\sim 40 \%$ and $\sim 30 \%$ in wt-PrP and Ifn $\gamma$-PrP expressing cells, respectively (Figure 1D). When cells expressing ER-GFP, PrP variants and cyPrP were immunostained with RTN4 antibody, Prl-PrP showed two distinct ER domains: ER-GFP labelled ER sheets and RTN4 labelled ER tubules. In cells expressing wt-PrP and Ifn $\gamma-\operatorname{PrP}$ this distinction was severely reduced (Supplementary Figure 2A and B). Similarly, fractionation of ER with iodixanol gradient showed presence of TRAP $\alpha$ (a protein of the translocon machinery to mark ER sheets), predominantly in the denser fractions in cells cotransfected with Prl-PrP and cyPrPRFP and RTN4 (a marker for tubular ER) was observed in the lighter fractions. However, this preferential partitioning of ER proteins was disrupted in the presence of wt-PrP and Ifn $\gamma$-PrP (Supplementary Figures 2C and 2D). In these fractions, TRAP $\alpha$ could be detected in the lighter fractions, while RTN4 was redistributed towards the denser fractions. Anomalous repartitioning of structural and functional proteins of the ER was more pronounced in the presence of Ifn $\gamma$-PrP than wt-PrP; the total levels of TRAP $\alpha$ and RTN4 did not significantly change due to the presence of the PrP variants (Supplementary Figure 2E). These data further suggest a plausible loss of ER architecture in cells with weaker signal sequences in presence of aggregation prone proteins — this is in turn suggestive of altered ER-homeostasis.

\section{Destabilisation of the actin network in cells with aggregation prone proteins}

Fragmentation and remodelling of the ER is often accompanied by changes in the actin cytoskeleton. While it is absolutely undisputed that microtubule (MT) network forms the major molecular tracks for ER tubule movement in animal cells, there is also ample evidence to suggest that the ER interacts with the actin cytoskeleton in differentiated animal cells. ${ }^{29-33}$ Importantly, actin-based mechanical linkages have been shown to enable ER spreading in mouse embryonic fibroblasts. ${ }^{34}$ Here, staining cells with Alexa-568 labelled Phalloidin showed long actin filaments in Prl-PrP expressing cells ( $85 \%)$ compared to a more punctuate staining pattern in wt-PrP $(\sim 67 \%)$ and Ifn $\gamma-\operatorname{PrP}(\sim 83 \%)$ expressing cells. This change in actin cytoskeleton is noted even when CFP tagged cyPrP is replaced by huntingtin aggregates $\left(\mathrm{Htt}_{\mathrm{Q} 103}-\mathrm{GFP}\right) .{ }^{35}$ Control cells transfected with empty vector did not show any change in actin cytoskeleton irrespective of the presence of PrP with different signal sequences - 90\% cells across samples had long actin filaments (Figures 2A and 2B). We next verified if the F/G actin ratio was affected under similar conditions since it is known across systems that 
disruption of actin polymerisation leads to remodelling of the ER network. ${ }^{34,36-39}$ There is a reduction in the $\mathrm{F} / \mathrm{G}$ actin ratio in samples with wt-PrP and Ifn $\gamma-\mathrm{PrP}$, suggesting a decrease in the amount of F-actin polymers. F/G actin ratio is similar in cells expressing Prl-PrP and the control with empty vector (Figure 2C). Ifn $\gamma$-PrP samples with the weakest signal sequence had the highest proportion of soluble actin - abundant presence of actin in this form is known to disrupt ER architecture. ${ }^{36}$

\section{Altered ER-Golgi trafficking in presence of nontranslocated proteins and and cytosolic aggregates}

Next, we wanted to see whether the change in ER morphology affected ER function. Hence, to verify whether the trafficking of proteins from ER to Golgi was affected in these cells, we co-immunostained the ER and Golgi using $\alpha$-TRAP $\alpha$ and $\alpha$-GM130 antibodies, respectively. The Golgi apparatus formed a characteristic ribbon situated next to the nucleus in cells cotransfected with GFP tagged cyPrP and Prl-PrP. Presence of wt-PrP and Ifn $\gamma$-PrP caused fragmentation of GM130 labelled Golgi (Figures 3A and 3C) as is identified by the wide spread of the compartment in the perinuclear area. In all the images the nuclei were stained with DAPI to better visualise the localisation of the Golgi apparatus. This observation was similar in cells containing Huntingtin aggregates ( $\mathrm{Htt}_{\mathrm{Q} 103}-\mathrm{GFP}$ ) (Figures $3 \mathrm{~B}$ and $3 \mathrm{C}$ ) where again discontinuous fragments of the Golgi widely spread out around the nucleus were most pronounced in Ifn $\gamma$-PrP samples. These results suggest that the presence of cytosolic aggregates and nontranslocated proteins cause disruption of ER structure along with fragmentation of Golgi.

Further, to check whether protein trafficking was affected in these cells with vacuolated ER and fragmented Golgi, we used a temperature-sensitive variant of vesicular stomatitis virus $G$ protein tagged with GFP (VSVG-GFP). ${ }^{40}$ This protein is restricted in the ER when cells are maintained at $40^{\circ} \mathrm{C}$ but is trafficked to the Golgi and plasma membrane once temperature is shifted to $32^{\circ} \mathrm{C}$. Differences in kinetics of trafficking were monitored in HeLa cells cotransfected with VSVG-GFP, RFP tagged cyPrP and PrP signal sequence variants. 48h after transfection, cells were transferred to $40^{\circ} \mathrm{C}$ and maintained for $16 \mathrm{~h}$. They were then transferred to $32^{\circ} \mathrm{C}$, fixed at different time points (0min, $7 \mathrm{~min}, 20 \mathrm{~min}$ and $30 \mathrm{~min}$ ) and cells were imaged by confocal imaging. In Prl-PrP expressing cells, VSVG-GFP was chiefly localised in the Golgi by 20min; it was detected also on the cell surface 30min after transfer to $32^{\circ} \mathrm{C}$ (Supplementary Figure $3 \mathrm{~A}$ ). An increase in the size of vesicles that ultimately lead to 
the formation of Golgi could be detected as early as 7min (Supplementary Figure 3B). In cells with wt-PrP, VSVG-GFP was primarily detected at the ER at $7 \mathrm{~min}$ and while at 20min the vesicular size was comparable to Prl-PrP vesicles at $7 \mathrm{~min}$. At 30min, however the vesicle sizes were comparable in cells with Prl-PrP and wt-PrP. In Ifn $\gamma$-PrP expressing cells, VSVGGFP was primarily in the ER till 30min. As in Figure 3, the tight Golgi cap near the nucleus that is seen in Prl-PrP expressing cells appears fragmented in wt-PrP and Ifn $\gamma$-PrP expressing cells (Supplementary Figure 3B).

\section{Aggregation proneness of reticulons facilitates disruption of ER}

It is known that while $\operatorname{PrP}_{40-231-m F P}$ forms intracellular aggregates, the different signal sequence bearing PrP variants used in our experiments do not form aggregates by themselves. ${ }^{9}$ These sequences were analysed with two algorithms-PASTA and TANGO that can predict aggregation tendencies of proteins from their sequences. ${ }^{41},{ }^{42}$ It was seen that RTNs have higher aggregation potential which makes it probable for them to adhere to preexisting cytosolic aggregates, leading to a disruption in the morphology of the ER. Also, both these algorithms indicate that presence of different signal sequences (Prl, PrP or Ifn $\gamma$ ) do not affect inherent aggregation tendency of PrP; they only determine the amount of nontranslocated PrP that is available to adhere to pre-existing aggregates. Cytosolic proteins like GAPDH, secreted proteins like Prolactin and Interferon- $\gamma$ have low aggregation tendencies (Supplementary Figure 4A and B).

Confocal laser scanning microscopy of N2A cells transfected with CFP tagged cyPrP and Ifn $\gamma$-PrP showed multiple aggregates in various sizes. Our analyses by immuno-electron microscopy (IEM) clearly indicated the presence of aggregates (marked by black arrowheads) less than $0.1 \mu \mathrm{m}$ (Supplementary Figure 4C). Curiously, ER was also detected in close proximity with these aggregates as marked (by red stars). It is hence logical to suggest that there are multiple smaller aggregates which also contribute to the destabilisation of the ER. Hela cells transfected with RFP tagged cyPrP, and different signal sequence bearing PrP variants were semi-permeabilised with digitonin to separate membrane and cytosolic fractions. It has been previously reported that cyPrP aggregates remain affixed in cells upon selective release of freely diffusible cytosolic contents $(C)$ by digitonin-mediated semipermeabilization of the plasma membrane. ${ }^{43}$ The remaining cellular components were extracted in a detergent containing buffer - two fractions were obtained, one detergent soluble (M) and another insoluble (I). RFP aggregates of cyPrP were noted chiefly in the 
detergent insoluble fraction by immunoblotting with RFP. RTN4 was primarily present in detergent soluble membrane fractions in all samples. However, an increasing amount of this reticulon family protein was detected in the detergent insoluble fractions in wt-PrP and Ifn $\gamma$ PrP expressing cells. This proportion was negligible in cells expressing Prl-PrP $(\leq 5 \%)$ but was $\sim 30 \%$ and $\sim 55 \%$ in wt-PrP and Ifn $\gamma$-PrP expressing cells, respectively (Figures $4 \mathrm{~A}$ and B). The total RTN4 protein across samples did not get altered significantly. A corresponding imaging experiment showed increased number of RTN4 positive aggregates in wtPrP and Ifn $\gamma$-PrP expressing cells compared to those with Prl-PrP. RTN4 was partially retained on aggregates in cells with wtPrP and Ifn $\gamma$-PrP even after semi-permeabilisation with digitonin (Figures 4C and D). These results suggested that aggregation proneness of RTN4 with cyPrP was enhanced in the presence of inefficient protein translocation; this raised the possibility that these observations with nontranslocated PrP might also apply to other proteins. Like before, we utilised the native G protein-coupled receptor (CRFR1) to test this hypothesis. In the presence of CRFR1, RTN4 was detected in $\sim 25 \%$ of cells with RFP tagged cyPrP. Importantly, replacing the signal sequence of CRFR1 with that from Prl (Prl-CRFR1) reduced the fraction of cells showing co-aggregation of RTN4 approximately threefold to $\sim 8 \%$. Semi-permeabilisation of cells with digitonin again reflected similar results (Figures 4E and F).

To establish this as a cell independent phenomenon, similar experiments were performed in SH-SY5Y cells. In these cells, we found that the proportion of RTN4 in the insoluble fraction was $\sim 10 \%$ in presence of Prl-PrP while it was $\sim 37 \%$ and $\sim 45 \%$ in samples with wt-PrP and Ifny-PrP, respectively (Supplementary Figures 5A and B). Imaging experiments showed that in $\sim 3 \%$ cells with Prl-PrP, the RTN4 positive aggregates could be detected while $\sim 33 \%$ and $\sim 39 \%$ wtPrP and Ifn $\gamma$-PrP expressing cells, respectively exhibited this phenotype (Supplementary Figures 5C and D). To extend this observation in a neuron-like cell system, SH-SY5Y cells were treated with all-trans retinoic acid (ATRA) to push them towards a neuronal fate; samples were analysed for the propensity of RTN4 to fractionate in the detergent insoluble fractions. Here again, an enrichment of RTN4 in the insoluble fractions was observed in samples with wt-PrP and Ifn $\gamma-\operatorname{PrP}$ (Supplementary Figures 5E and F). Imaging of digitonin semi-permeabilised cells showed increased number of RTN4 positive aggregates in presence of wtPrP and Ifn $\gamma$-PrP as compared to those with Prl-PrP (Supplementary Figures 5G and $\mathrm{H}$ ). 
To further validate that RTN4 was sequestered on cytosolic aggregates, SH-SY5Y cells transfected with RFP tagged cyPrP, different signal sequence bearing PrP variants and RTN4-GFP were imaged in real-time. Semi-permeabilisation with digitonin did not affect the reticular expression pattern of RTN4 across the samples (Supplementary Figure 6A). Addition of membrane extraction buffer to these samples showed retention of RTN4-GFP signal on RFP tagged aggregates in cells with wt-PrP; the RTN4-GFP signal retained on RFP tagged aggreagates was more in the presence of Ifn $\gamma-\operatorname{PrP}$, when cells were imaged with same parameters (Supplementary Figures 6A and 6B). The results so far indicate that sequestration of this ER shaping protein to cytosolic aggregates in presence of nontranslocated proteins emerges as one of the key factors causing alterations in ER morphology. This is a general phenomenon occurring in multiple cell types and in presence of different nontranlocated precursor proteins. While RTN4 could be detected on aggregates, the fraction of this reticulon protein contributing towards this could be either membrane-inserted or a population that failed to be correctly inserted. Further experiments would be required to correctly identify the origin of the RTN4 fraction that co-aggregates with cyPrP.

\section{Presence of nontranslocated proteins collapse three-way ER junctions}

The repartitioning of ER compartments and sequestration of RTN4 into cytosolic aggregates led us to hypothesize that the stability of three-way junctions would be compromised in presence of nontranslocated precursor proteins. To verify this, Hela cells cotransfected with KDEL-mRuby, GFP tagged cyPrP and different signal sequence bearing PrP variants were imaged in live for $5 \mathrm{~min}$ - the time required for the junctions to break and reform was the fastest in cells with Prl-PrP and slowest in those with Ifn $\gamma$-PrP. The panel with enlarged views of the three-way junctions clearly showed a loss in their structural integrity in cells with wt-PrP and Ifn $\gamma$-PrP (Figure 5). Presence of nontranslocated proteins led to more than three folds decrease in the number of the junction in $100 \mu \mathrm{m}^{2}$ area, thus indicating a loss of stability of the three-way junctions and discontinuity of the ER network. It is suggested that Lunapark (LNPK), a member of the conserved Lunapark protein family, is required for the maintenance of the ER network in yeast and mammalian cells. ${ }^{44-46}$ Cells transfected as before were immunostained against LNPK and imaged. Snapshots of these images show a significant decrease in the number of LNPK positive RTN4 junctions at the ER (Figure 6A and C). Further, depletion of LNPK is known to shift the balance of ER from tubular to more sheet-like morphology. ${ }^{46}$ In this study we found that an abundance of nontranslocated 
proteins led to an increase in the percentage of cells that show association between LNPK and the ER sheet marker SEC61- $\beta$, and a simultaneous decrease in the three-way junction protein and the tubule marker RTN4 (Figure 6B and C). These together imply a relative shift in the overall ER sheet to tubule ratio in the presence of cytosolic aggregates and nontranlocated proteins (Figure 6D).

Cumulatively, our results show that cytosolic aggregates together with a heavy load of nontranslocated proteins are indeed detrimental to the health of the ER - the distribution of the ER compartments is disrupted at the structural level and this is reflected as compromised protein trafficking at the functional level.

\section{Discussion}

This study shows an important effect of increase in the load of nontranslocated proteins in the cell. When there is a combination of nontranslocated proteins and cytosolic protein aggregates, the ER morphology and dynamics is perturbed leading to vacuolation, fragmentation of the ER and disruption of the three-way junctions. In cells expressing wtPrP/ Ifn $\gamma$-PrP and cyPrP (that forms cytosolic aggregates), the Golgi loses its tight perinuclear-capped organisation and appears fragmented. Protein trafficking from the ER to Golgi is affected in these cells. Here, we further show that a possible contributing factor to the breakdown of ER membranes is the propensity of RTN proteins to be attracted to cytosolic protein aggregates that persist and grow in presence of nontranslocated proteins (Figure 7).

This study is significant because protein aggregates are an integral part of a number of neurodegenerative diseases. These aggregates alter cellular homeostasis in multiple ways including quality control pathways, intracellular transport, lysosomal function, and ROS generation. Aggregates often sequester essential cellular components leading to functional depeletion of several proteins. For example cyPrP interacts with the E3 ligase Mahogunin Ring Finger 1 leading to its sequestration. ${ }^{43}$ Sequestration of MGRN1 in turn leads to decreased ubiqutination of its substrate GP78. ${ }^{47}$ Huntingtin aggregates affect CBP (CREB binding protein) mediated transcription by sequestering $\mathrm{CBP}$ in aggregates. ${ }^{48}$ Amyloid protein aggregates have been shown to interact with numerous cellular proteins, some of 
which are in hub positions of important protein-protein interaction networks. These interactors are often unstructured, an important feature of moonlighting proteins that have numerous, often unrelated functions. ${ }^{10}$

Membrane damage is an important feature of neurodegenerative diseases. Amyloid beta protein plaques form channels in neuron cell membranes in AD. ${ }^{49}$ ER stress has been noted in several diseases like PD, AD and ALS. ${ }^{50}$ This often impairs protein trafficking and causes build up of ERAD substrates. Golgi fragmentation has been seen in ALS and AD. ${ }^{51,52}$ The direct effect of protein aggregates on biological membranes has not been deciphered in in vivo systems. In vitro experiments with amyloid fibrils and artificial membrane matrices show that lipid-fibril interactions can affect formation of biological membranes. ${ }^{53}$ It has been recently reported that Shadoo (Sho), a patially proteinase K (PK)-resistant and aggregation prone member of prion protein family, associates with lipid rafts and some of it can be found to localise at the ER. ${ }^{54}$ Therefore, it will be interesting to see how cytosolic aggregates can affect lipid composition of different endomembranes and how this is affected by the presence of nontranslocated proteins.

In this study, we show the presence of reticulons in cytosolic aggregates - this can directly contribute to membrane fragmentation as noted in cells with cyPrP and wt-PrP/Ifn $\gamma-\operatorname{PrP}$. Recent studies show that the reticulon family of proteins maintain ER shape and curvature, but each of these family members has specific functions like ER turnover, dendritic remodelling and membrane trafficking. ${ }^{55,56}$ It is reported that loss of a single isoform of RTN4, RTN4b, is sufficient to alter ER morphology due to loss of ER tubulation and redistribution of STIM1 to ER sheets. ${ }^{57}$ Further, RTN4 proteins, NOGOB/RTN4B and NOGO-A/RTN4A localize preferably to curved membranes on ER tubules and sheet edges. They are known to be involved in forming and maintaining ER tubules as their over-expression alters the balance strongly towards tubules and causes the deformation of the cell shape. On the contrary, depletion of RTN4 proteins induces formation of large peripheral ER sheets. ${ }^{58}$ Therefore, it is plausible to suggest that functional depletion of reticulons from the ER in aggregate containing cells can affect ER structure and other aspects of cellular physiology where their functions are essential. Presence of nontranslocated proteins increases persistence of cytosolic aggregates and may even promote their growth by co-accumulation of some of the ER-shaping proteins. This was supported by the increase in 
RTN4 in detergent insoluble fractions in cells expressing PrP with the weak signal sequences (wt-PrP and Ifn $\gamma$-PrP).

It is plausible to extrapolate that depletion of these ER-shaping proteins would trigger a feedback signal to further destabilise this organelle. There are evidences in the literature to suggest that while depletion of the reticulon, FAM134B leads to ER expansion of the sheets, ${ }^{21}$ and a compromise in RTN3 activity affects the ER tubules. ${ }^{22}$ Hence, sequestration or functional depletion of these structural proteins of the ER would skew the relative tubulesheet balance. In the presence of cytosolic aggregates, abundance of nontranslocated proteins similarly affects the sub-compartments of the ER - altered partitioning of the tubule and sheet markers are seen in presence of proteins with weaker signal sequences - all indicative of ER membrane remodelling (EMR).

The data presented here - based on the mobility of ER-GFP, the differential distribution of ER sheets and tubule marker proteins and compromised trafficking of VSVG-GFP - all indicate EMR in presence of nontranslocated precursor proteins. There is an intimate association of Golgi bodies with moving ER exit sites $^{59}$ and ER tubule growth appears to follow Golgi body movement ${ }^{60}$ in plant systems. EMR affects ER to Golgi network (anterograde) protein trafficking; conversely, inhibition of anterograde trafficking also leads to EMR. ${ }^{61,62}$ Thus inhibition of protein transport from ER to Golgi as is detected when nontranslocated proteins are in abundance could be an important contributing factor in EMR. Endoplasmic reticulum exit sites (ERES) are present on the high curvature tubular ER membranes. ${ }^{63}$ Altered distribution of tubular ER would affect the number of ERES on the ER membrane and eventually result in delayed protein transport out of the ER. Further, it is reported that depletion of the integral ER-membrane proteins, VAP-A and VAP-B, affect the structural and functional integrity of the Golgi complex with a decrease in Golgi to plasma membrane protein transport. ${ }^{64}$ Our study also reports a similar destabilisation of the Golgi complex and delayed protein transport to the plasma membrane.

It is implied that destabilisation of the actin cytoskeleton affects the tubule-sheet distribution $^{34,38,39,65}$ and also leads to generation of areas void of ER in the cell periphery. Like actin, LNPK plays a crucial role in maintaining the ER network; rather LNPK is needed to stabilize the actin-dependent remodelling of the ER. ${ }^{45,66}$ LNPK is important for stabilising nascent three-way ER junctions and its loss of function leads to collapse of the extended ER network into a more compact, sheet-like structure. Cooperation between the ER-shaping 
proteins like LNPK and reticulons is required to generate a tubular membrane network and tubule to sheet conversion of the ER. ${ }^{67}$ Presence of aggregation-prone nontranslocated precursor proteins $\left[{ }^{9}\right]$ destabilises the three-way junctions and collapses the tubular network.

While the effect of cytosolic aggregates on cellular physiology has been studied in detail, little is understood about the pathway of formation of aggregates and the dynamics of their growth. Nontranslocated proteins may be an important factor in determining the cytotoxic nature of aggregates as seen by their effect on ER membranes due to functional loss of essential but unrelated proteins. ${ }^{9}$ It will be also interesting to see how other endomembranes like that of the mitochondria, peroxisomes behave in presence of nontranslocated proteins and cytosolic aggregates. Presence of nontranslocated proteins may affect other aggregate mediated pathophysiology like intracellular transport of protein cargo, lysosomal function, and mitochondrial ATP synthesis. 


\section{Materials and methods}

\section{Constructs, antibodies and reagents}

wt-PrP, Prl-PrP, Ifn $\gamma$-PrP, CRFR1, Prl-CRFR1 constructs have been described before. ${ }^{9,13}$ These constructs have the PrP gene from Syrian Hamster (Mesocricetus auratus) cloned with its wt signal sequence at the $\mathrm{N}$ terminal (MANLSYWLLALFVAMWTDVGLC), or with a bovine prolactin signal sequence (MDSKGSSQKGSRLLLLLVVSNLLLCQGVVS) or with the signal sequence of Pig Interferon- $\gamma$ (MSYTTYFLAFQLCVTLC). mFP-PrP $40-231$ and GFP tagged $\mathrm{Htt}_{\mathrm{pQ} 103}$ have been described before. ${ }^{9}$ ER-GFP was a gift of Erik L. Snapp. VSVGGFP was a gift from Jennifer Lippincott-Schwartz. RTN4-GFP and sec61- $\beta$-GFP were gifts of Rafael Mattera.

Antibodies were from the following sources: RTN4 (Abcam, Cambridge, UK), GM130 (Abcam, Cambridge), LNPK (Abcam, Cambridge), Nogo A+B [RTN4] (Abcam, Cambridge), C4 actin (Abcam, Cambridge). The RFP, GFP, TRAP $\alpha, 3 F 4$ antibodies were gifts of Ramanujan S Hegde (Cambridge, UK).

Regeants were from the following sources: MG132 (Sigma Aldrich), ATRA (SigmaAldrich), digitonin (Sigma-Aldrich), cytochalasin D (Sigma Aldrich), Optiprep (Sigma Aldrich), TCA (Merck), Alexa568-labeled Phalloidin (\#A-12380; ThermoFischer Scientific).

\section{Cell culture, transfection and ATRA treatment}

Maintenance of cells in culture was as before. ${ }^{9,68} \mathrm{SH}-\mathrm{SY} 5 \mathrm{Y}$ cells were cultured in high glucose $(4.5 \mathrm{~g} / \mathrm{l})$ DMEM. They were subjected to $10 \mu \mathrm{M}$ ATRA treatment for 5 days during 
differentiation studies. ${ }^{69,70}$ For imaging experiments, cells subjected to ATRA treatment were semi-permeabilised with digitonin or without semi-permeabilisation were fixed and imaged. Briefly, cells were grown in 10\% fetal bovine serum (FBS; Gibco, Grand Island, NY, USA)/Dulbecco's modified Eagle's medium (DMEM; Himedia, Mumbai, India) at $37^{\circ} \mathrm{C}$ and $5 \% \mathrm{CO}_{2}$. For transfections of cells, Lipofectamine 2000 was used (Invitrogen, Carlsbad, CA, USA) as per the manufacturer's instructions. All tissue culture plastic-ware and Lab-Tek 8well chambered slides used for microscopy were from Nunc, bottom coverglass dishes used for microscopy were from SPL Lifesciences.

\section{Immunocytochemistry}

For immunocytochemistry, cells were fixed with either $4 \%$ formaldehyde or methanol as per the requirement of the antibody, like before. ${ }^{68,43}$ Cells were permeabilized using $10 \%$ FBS, PBS and $0.1 \%$ saponin (Sigma-Aldrich) for $60 \mathrm{~min}$, followed by overnight staining in primary antibody at $4^{\circ} \mathrm{C}$ and $60 \mathrm{~min}$ incubation in secondary antibody at room temperature. The samples were then imaged using confocal microscopy using the Zeiss LSM 510 and Nikon A1R+ Ti-E with N-SIM and FCS microscope systems.

\section{Confocal imaging and image analyses}

Confocal imaging was done using Zeiss LSM 510/710 and the Nikon A1R+ Ti-E with NSIM and FCS microscope system. Ar-ion laser (for GFP excitation or Alexa-Fluor 488 with the $488 \mathrm{~nm}$ line), a He-Ne laser (for RFP, Alexa-Fluors 594 excitation with the 561 line were used with $63 \mathrm{X}$ or $100 \times 1.4$ NA oil immersion objective. Actin filaments were visualised with Alexa568 labelled Phalloidin (ThermoFischer Scientific, \#A-12380, Eugene, OR, USA) using standard protocols.

For quantitative analyses of ER vacuolation, images were converted to 8-bit grayscale binary format. These were then thresholded and skeletonised in FIJI. Two ROIs of $25 \mu \mathrm{m}^{2}$ were selected per cell. ER fragments were counted and any cell with more than 10 fragments in an ROI was classified as having "fragmented ER". 80 cells per set from over 3 independent experiments were analysed by two individuals; any cell scored by both individuals as having "fragmented ER" was taken for plotting.

Image analyses to quantify colocalisation between RTN4 and cyPrP were done using Coloc2 plugin in FIJI. At least 60 aggregates taken from 30 cells over 3 independent experiments 
were analysed to calculate percentage of RTN4 positive aggregates. ER fusion events were quantified as described previously. ${ }^{71}$ For this, the number of tubular ER extension events that occurred in $10 \mu \mathrm{m}^{2}$ ROIs over a $5 \mathrm{~min}$ time course was scored as either resulting in a successful or unsuccessful fusion event. The percentage of successful fusion events over total number of events was plotted. 100 ROIs from 30 cells per set, taken from 3 independent experiments were analysed.

For quantitative analyses of vesicles culminating in the Golgi, 600 X 600 pixel $(\sim 39 \mu \mathrm{m} \mathrm{X}$ $\sim 39 \mu \mathrm{m}$ ) confocal images were collected where each pixel represents an area of $\sim 0.0042 \mu \mathrm{m}^{2}$. All images were processed via implementation of morphological operations using MATLAB image processing tool box. ${ }^{72}$ Vesicular structures were extracted out by setting a dynamic intensity cut-off (range: 100-150) with respect to the overall brightness of the individual image. Morphological operations like erosion and dilation were used to separate out overlapping vesicular cluster. bwareaopen function was used to filter out smaller noise like regions $\left(<=0.042 \mu \mathrm{m}^{2}\right)$ whereas regionprops was used to estimate the area of the vesicles. GraphPad Prism was used to plot the distribution of area of the vesicles.

\section{FRAP assay}

HeLa cells transfected with cyPrP, PrP variants and ER-GFP were subjected to FRAP assay $48 \mathrm{~h}$ after transfection. $5 \mu \mathrm{m}^{2}$ ROIs were marked followed by photobleaching using $75 \%$ Argon laser power for 10 s followed by time-lapse imaging for $4 \mathrm{~min}$ at continuous mode at 5fps. \% recovery after photobleaching was plotted in MS Excel after measuring signal intensity of ROIs by FIJI.

\section{Western blotting}

The protocol for western blotting was as described before. ${ }^{68} 10 \%$ Tris-tricine gels were used for SDS-PAGE followed by Western blotting. Quantification of Western blots was done using Quantity One software of Bio-rad. At least 3 independent experiments were performed and band intensities were normalized to loading control.

\section{VSVG-GFP protein trafficking assay}

HeLa cells transfected with VSVG-GFP, cyPrP and PrP variants were transferred to $40^{\circ} \mathrm{C}$ $48 \mathrm{~h}$ after transfection. After $16 \mathrm{~h}$ they were transferred to $32^{\circ} \mathrm{C}$, and fixed at different time 
points. VSVG-GFP was imaged by confocal imaging or samples were lysed in $1 \mathrm{X}$ NEB EndoH denaturing buffer for EndoH assay.

\section{EndoH sensitivity assay}

Lysates were made in 1X NEB denaturing buffer (5\% SDS and 10\% 2-mercatptoethnaol) for $5 \mathrm{~min}$ at $100^{\circ} \mathrm{C}$, cooled and mixed with $1 / 10$ concentrated EndoH reaction buffer (G5: $0.5 \mathrm{M}$ sodium citrate, $\mathrm{pH} 5.5$ ). These were then digested with 500units of EndoH for $5 \mathrm{~h}$ at $37^{\circ} \mathrm{C}$. Sample buffer was added to stop the reaction. Samples were boiled and loaded onto SDS-PAGE followed by immunoblotting using GFP antibodies. Lysates prior to EndoH digestion were used as loading control.

\section{Digitonin semi-permeabilisation of cells}

Biochemical fractionation was similar to previous methods. ${ }^{15}$ Cells were washed with $2 \mathrm{ml}$ KHM [20mM HEPES (pH 7.4), $110 \mathrm{mM}$ potassium acetate, $2 \mathrm{mM} \mathrm{MgCl}$ ] and extracted with KHM containing digitonin (at $100 \mu \mathrm{g} / \mathrm{ml}$ ) to generate the soluble cytosolic fraction (C). The cells were then washed once in KHM and subsequently extracted in $1 \mathrm{ml}$ buffer $[50 \mathrm{mM}$ HEPES ( $\mathrm{pH} 7.4$ ), $150 \mathrm{mM} \mathrm{NaCl}, 0.1 \%$ SDS, $0.5 \%$ sodium deoxycholate and protease inhibitor cocktail] to recover the detergent-soluble wash fraction primarily containing the membranes (M). The remaining insoluble material (sedimented for $15 \mathrm{~min}$ in a microfuge) at $15000 \mathrm{~g}$ (I) was dissolved in 1\% SDS. Proteins from the soluble and wash fractions were precipitated using 12\% Trichloroacetic acid, washed in acetone, and dissolved in SDS prior to analysis. Fractions were loaded onto SDS-PAGE and immunoblotted using RTN4 and RFP antibodies. For analysis of the total protein content, equivalent proportions from C, M \& I fractions were mixed and subjected to Western blotting. GAPDH was used a loading control.

For confocal imaging, cells were treated with KHM buffer containing $100 \mu \mathrm{g} / \mathrm{ml}$ digitonin for 5 min followed by fixing cells with $4 \%$ formaldehyde, blocking in solution containing PBS, $10 \%$ FBS, $0.1 \%$ saponin; the samples were then immunostained for RTN4.

\section{Semi-permeabilisation and membrane extraction for real-time imaging}

For imaging interactions between proteins randomly chosen fields of cells were rinsed with $2 \mathrm{ml} \mathrm{KHM}$ and then put into $1 \mathrm{ml} \mathrm{KHM}$ to capture pre-digitonin treatment images. The KHM was replaced with $1 \mathrm{ml} \mathrm{KHM}$ containing $100 \mu \mathrm{g} / \mathrm{ml}$ digitonin and imaged at $1 \mathrm{~min}$ interval for a total of 10min. After this the KHM-digitonin buffer was removed and replaced by the 
detergent containing membrane extraction buffer. Images were taken at $1 \mathrm{~min}$ interval for 3 min. 3 dishes were imaged for each set of transfections and the complete set of experiments was performed 3 times to verify the results.

\section{Immunoprecipitation with detergent insoluble pellet fraction}

HeLa cells transfected with Ifn $\gamma-\operatorname{PrP}$ and cy-PrP aggregate were semi-permeabilized using KHM-digitonin buffer as before. The soluble cytosolic (C) and the detergent soluble membrane extracted fractions (M) were discarded. The detergent insoluble pellet (I) was resuspended in $50 \mu 1$ buffer ( $0.5 \mathrm{M}$ Tris-pH 6.8, 10\% SDS, $\beta$-mercapto-ethanol), boiled for 10 minutes and cooled down to room temperature. To this $1 \mathrm{ml}$ IP buffer $[50 \mathrm{mM}$ Tris- $\mathrm{HCl}(\mathrm{pH}$ 7.5), $150 \mathrm{mM} \mathrm{NaCl}, 0.1 \%$ Triton-X, $1 \%$ NP-40] followed by immunoprecipitation and immunoblotting with the indicated antibody pairs.

\section{F/G actin fractionation}

Cells were washed once in ice-cold PBS before lysis with actin stabilization buffer $(0.1 \mathrm{M}$ PIPES, pH6.9, 30\% glycerol, 5\% DMSO, 1mM MgSO 4 , 1mM EGTA, 1\% TX-100, 1mM ATP, and protease inhibitor) on ice for $10 \mathrm{~min}$. Cells were dislodged by scraping and the entire extract centrifuged at $4^{\circ} \mathrm{C}$ for $75 \mathrm{~min}$ in a tabletop centrifuge at $16000 \mathrm{~g}$. The supernatant containing G-actin was recovered, and the pellet containing F-actin was solubilised with actin depolymerization buffer (0.1M PIPES, pH6.9, $1 \mathrm{mM} \mathrm{MgSO} 4,10 \mathrm{mM}$ $\mathrm{CaCl}_{2}$, and $5 \mu \mathrm{M}$ cytochalasin D). Equal amount of both suspensions were separated on $12 \%$ SDS-PAGE gels and then western blotted with the C4 actin antibody. Optical band density was analysed as indicated above to estimate the cellular F/G-actin ratio.

\section{Fractionation of ER fractions using optiprep}

Five $60 \mathrm{~mm}$ plates of HeLa cells were lysed in isolation buffer [50mM HEPES-KOH $(\mathrm{pH}$ 7.4), 200mM sucrose, $1 \mathrm{mM}$ EGTA, protease inhibitor cocktail] by syringe lysis. Nucleus was removed by centrifugation at $1500 \mathrm{~g}$ for $5 \mathrm{~min}$. The postnuclear supernatant was centrifuged at $10,000 \mathrm{~g}$ for $1 \mathrm{~min}$ to remove mitochondria. The supernatant was centrifuged at $1,00,000 \mathrm{~g}$ for $1 \mathrm{hr}$ to obtain the microsomal pellet, which was then diluted with OptiPrep (60\% solution from Millipore Sigma) to a final concentration of 20\%. A $12 \mathrm{ml}$ OptiPrep gradient was prepared by layering $3 \mathrm{ml}$ of $30 \%$ OptiPrep, $3 \mathrm{ml}$ sample in $20 \%$ OptiPrep followed by $6 \mathrm{ml}$ of 
$15 \%$ OptiPrep. This was then ultracentrifuged at 1,50,000g for $3 \mathrm{~h}$. Two rings were observed. The SER forms a ring just below the surface, whereas the RER forms a ring at the middle of the gradient. Fractions of $1 \mathrm{ml}$ were collected from the bottom upward, followed by TCA precipitation. The TCA pellet was mixed with sample buffer followed by immunoblotting with TRAP $\alpha$ and RTN4 antibodies. RTN4 served as marker for the SER (ER tubules) and TRAP $\alpha$ served as marker for RER (ER sheets).

\section{Immuno-electron microscopy (IEM)}

Cells were prepared for IEM as described previously. ${ }^{73}$ Briefly, cells were fixed in $4 \%$ paraformaldehyde, and then blocked with $1 \%$ bovine serum albumin in PBS. Cells were permeabilized and incubated with primary GFP antibody (rabbit anti-GFP; Molecular Probes) and subsequently incubated with nanogold-conjugated secondary antibodies (Nanoprobes). Cells were fixed with glutaraldehyde, treated with gold enhancement mixture for $6 \mathrm{~min}$ and postfixed in reduced osmium prior to embedding in Epon. 70-100nm sections were cut and stained with lead citrate prior to imaging.

\section{Computational prediction of aggregation tendency}

Amino acid sequences of the mentioned proteins were retrieved from the UniProtKB ${ }^{74}$ and were submitted to two different algorithms, PASTA and TANGO to check for their propensity to aggregate. PASTA 2.0 implements a statistical energy function in order to determine fibril formation whereas TANGO implements an algorithm designed to predict cross-beta aggregation in peptides and proteins. Best energy from PASTA $2.0^{41}$ and aggregation tendency from $\mathrm{TANGO}^{42}$ were plotted as a measure of inherent aggregation propensity of the selected proteins. 
Author Contributions: PM, RM, DM and OC conceived the project. PM, RM, DM, ZK and DC primarily designed the experiments with suggestions from OC. RM, DM and OC interpreted the results and wrote the paper. SC performed the bioinformatics analyses, SD and SC performed quantitative imaging analyses for measuring vesicular area, IEM was done by RS.

Acknowledgements: We thank R.S.Hegde, J. Lippincott-Schwartz, R. Mattera and E.L.Snapp for plasmids; R. S. Hegde for antibodies; OC laboratory members for their help and support throughout the study. OC is supported by the "Integrative Biology on Omics Platform Project", intramural funding of the Department of Atomic Energy (DAE), Government of India. OC is partially funded by SERB, Department of Science \& Technology (EMR/2016/002706), Government of India. DM acknowledges financial support from University Grants Commission (D. M.; F2-32/1998 (SA-1). SC acknowledges CSIR-IICB for infrastructural support.

Conflict of interest: The authors declare no conflict of interest, financial or otherwise. 


\section{References}

1. Ross, C.A., Poirier, M.A. Protein aggregation and neurodegenerative disease. Nature Medicine. 2004; 10(7):S10.

2. Kopito, R.R. Aggresomes, inclusion bodies and protein aggregation. Trends in Cell Biology. 2000; 10(12):524-30.

3. Bence, N.F., Sampat, R.M., Kopito, R.R. Impairment of the ubiquitin-proteasome system by protein aggregation. Science. 2001; 292(5521):1552-5.

4. Hirabayashi, M., Inoue, K., Tanaka, K., et al. VCP/p97 in abnormal protein aggregates, cytoplasmic vacuoles, and cell death, phenotypes relevant to neurodegeneration. Cell Death and Differentiation. 2001; 8(10):977.

5. Sergeant, N., Wattez, A., Galvn-valencia, M., et al. Association of ATP synthase $\alpha$-chain with neurofibrillary degeneration in Alzheimer's disease. Neuroscience. 2003; 117(2):293303.

6. Pankiv, S., Clausen, T.H., Lamark, T., et al. p62/SQSTM1 binds directly to Atg8/LC3 to facilitate degradation of ubiquitinated protein aggregates by autophagy. Journal of Biological Chemistry. 2007; 282(33):24131-45.

7. Kaganovich, D., Kopito, R., Frydman, J. Misfolded proteins partition between two distinct quality control compartments. Nature. 2008; 454(7208):1088.

8. Woerner, A.C., Frottin, F., Hornburg, D., et al. Cytoplasmic protein aggregates interfere with nucleocytoplasmic transport of protein and RNA. Science. 2016; 351(6269):173-6.

9. Chakrabarti, O., Rane, N.S., Hegde, R.S. Cytosolic aggregates perturb the degradation of nontranslocated secretory and membrane proteins. Molecular Biology of the Cell. 2011; 22(10):1625-37.

10. Olzscha, H., Schermann, S.M., Woerner, A.C., et al. Amyloid-like aggregates sequester numerous metastable proteins with essential cellular functions. Cell. 2011; 144(1):67-78. 
11. Hegde, R.S., Voigt, S., Lingappa, V.R. Regulation of protein topology by trans-acting factors at the endoplasmic reticulum. Molecular Cell. 1998; 2(1):85-91.

12. Nilsson, I., Lara, P., Hessa, T., Johnson, A.E., von Heijne, G., Karamyshev, A.L. The code for directing proteins for translocation across ER membrane: SRP cotranslationally recognizes specific features of a signal sequence. Journal of Molecular Biology. 2015; 427(6):1191-201.

13. Kim, S.J., Mitra, D., Salerno, J.R., Hegde, R.S. Signal sequences control gating of the protein translocation channel in a substrate-specific manner. Developmental Cell. 2002; 2(2):207-17.

14. Rane, N.S., Kang, S.W., Chakrabarti, O., Feigenbaum, L., Hegde, R.S. Reduced translocation of nascent prion protein during ER stress contributes to neurodegeneration. Developmental Cell. 2008; 15(3):359-70.

15. Levine, C.G., Mitra, D., Sharma, A., Smith, C.L., Hegde, R.S. The efficiency of protein compartmentalization into the secretory pathway. Molecular Biology of the Cell. 2005; 16(1):279-91.

16. Kang, S.W., Rane, N.S., Kim, S.J., Garrison, J.L., Taunton, J., Hegde, R.S. Substratespecific translocational attenuation during ER stress defines a pre-emptive quality control pathway. Cell. 2006; 127(5):999-1013.

17. Karamyshev, A.L., Patrick, A.E., Karamysheva, Z.N., et al. Inefficient SRP interaction with a nascent chain triggers a mRNA quality control pathway. Cell. 2014; 156(1):146-57.

18. Voeltz, G.K., Prinz, W.A., Shibata, Y., Rist, J.M., Rapoport, T.A. A class of membrane proteins shaping the tubular endoplasmic reticulum. Cell. 2006; 124(3):573-86.

19. Shibata, Y., Hu, J., Kozlov, M.M., Rapoport, T.A. Mechanisms shaping the membranes of cellular organelles. Annual Review of Cell and Developmental. 2009; 25:329-54.

20. Yang, Y.S., Strittmatter, S.M. The reticulons: a family of proteins with diverse functions. Genome Biology. 2007; 8(12):234.

21. Khaminets, A., Heinrich, T., Mari, M., et al. Regulation of endoplasmic reticulum turnover by selective autophagy. Nature. 2015; 522(7556):354. 
22. Grumati, P., Morozzi, G., Hölper, S., et al. Full length RTN3 regulates turnover of tubular endoplasmic reticulum via selective autophagy. eLife. 2017; 6:e25555.

23. Kume, H., Konishi, Y., Murayama, K.S., Kametani, F., Araki, W. Expression of reticulon 3 in Alzheimer's disease brain. Neuropathology and Applied Neurobiology. 2009; 35(2):17888.

24. Heath, J.E., Siedlak, S.L., Zhu, X., et al. Widespread distribution of reticulon-3 in various neurodegenerative diseases. Neuropathology. 2010; 30(6):574-9.

25. Yang, Y.S., Harel, N.Y., Strittmatter, S.M. Reticulon-4A (Nogo-A) redistributes protein disulfide isomerase to protect mice from SOD1-dependent amyotrophic lateral sclerosis. Journal of Neuroscience. 2009; 29(44):13850-9.

26. Chen, R., Jin, R., Wu, L., et al. Reticulon 3 attenuates the clearance of cytosolic prion aggregates via inhibiting autophagy. Autophagy. 2011; 7(2):205-16.

27. Rane, N.S., Chakrabarti, O., Feigenbaum, L., Hegde, R.S. Signal sequence insufficiency contributes to neurodegeneration caused by transmembrane prion protein. The Journal of Cell Biology. 2010; 188(4):515-26.

28. Snapp, E.L., Sharma, A., Lippincott-Schwartz, J., Hegde, R.S. Monitoring chaperone engagement of substrates in the endoplasmic reticulum of live cells. Proceedings of the National Academy of Sciences. 2006; 103(17):6536-41.

29. Baumann, O. Structural interactions of actin filaments and endoplasmic reticulum in honeybee photoreceptor cells. Cell and tissue research. 1992; 268(1):71-9.

30. Sturmer, K., Baumann, O., Walz, B. Actin-dependent light-induced translocation of mitochondria and ER cisternae in the photoreceptor cells of the locust Schistocerca gregaria. Journal of cell science. 1995; 108(6):2273-83.

31. Wang, Y., Mattson, M.P., Furukawa, K. Endoplasmic reticulum calcium release is modulated by actin polymerization. Journal of neurochemistry. 2002; 82(4):945-52.

32. Poteryaev, D., Squirrell, J.M., Campbell, J.M., White, J.G., Spang, A. Involvement of the actin cytoskeleton and homotypic membrane fusion in ER dynamics in Caenorhabditis elegans. Molecular biology of the cell. 2005; 16(5):2139-53.b 
33. Wagner, W., Brenowitz, S.D., Hammer III, J.A. Myosin-Va transports the endoplasmic reticulum into the dendritic spines of Purkinje neurons. Nature cell biology. 2011; 13(1):40.

34. Lynch, C.D., Gauthier, N.C., Biais, N., et al. Filamin depletion blocks endoplasmic spreading and destabilizes force-bearing adhesions. Molecular biology of the cell. 2011; 22(8):1263-73.

35.Krobitsch, S., Lindquist, S. Aggregation of huntingtin in yeast varies with the length of the polyglutamine expansion and the expression of chaperone proteins. Proceedings of the National Academy of Sciences. 2000; 97(4):1589-94.

36. Sparkes, I., Runions, J., Hawes, C., Griffing, L. Movement and remodeling of the endoplasmic reticulum in nondividing cells of tobacco leaves. The Plant Cell. 2009; 21(12):3937-49.

37. Cao, P., Renna, L., Stefano, G., Brandizzi, F. SYP73 anchors the ER to the actin cytoskeleton for maintenance of ER integrity and streaming in Arabidopsis. Current Biology. 2016; 26(23):3245-54.

38. Joensuu, M., Belevich, I., Rämö, O., et al. ER sheet persistence is coupled to myosin 1cregulated dynamic actin filament arrays. Molecular biology of the cell. 2014; 25(7):1111-26.

39. Wales, P., Schuberth, C.E., Aufschnaiter, R., et al. Calcium-mediated actin reset (CaAR) mediates acute cell adaptations. Elife. 2016; 5:e19850.

40. Presley, J.F., Cole, N.B., Schroer, T.A., Hirschberg, K., Lippincott- Schwartz, J. ER-toGolgi transport visualized in living cells. Nature. 1997; 389(6646):81.

41. Walsh, I., Seno, F., Tosatto, S.C., Trovato, A. PASTA 2.0: an improved server for protein aggregation prediction. Nucleic Acids Research. 2014; 42(W1):W301-7.

42. Fernandez-Escamilla, A.M., Rousseau, F., Schymkowitz, J., Serrano, L. Prediction of sequence-dependent and mutational effects on the aggregation of peptides and proteins. Nature Biotechnology. 2004; 22(10):1302.

43. Chakrabarti, O., Hegde, R.S. Functional depletion of mahogunin by cytosolically exposed prion protein contributes to neurodegeneration. Cell. 2009; 137(6):1136-47. 
44. Chen, S., Novick, P., Ferro-Novick, S. ER network formation requires a balance of the dynamin-like GTPase Sey1p and the Lunapark family member Lnp1p. Nature Cell Biology. 2012; 14(7):707.

45. Chen, S., Desai, T., McNew, J.A., Gerard, P., Novick, P.J., Ferro-Novick, S. Lunapark stabilizes nascent three-way junctions in the endoplasmic reticulum. Proceedings of the National Academy of Sciences. 2015; 112(2):418-23.

46. Shemesh, T., Klemm, R.W., Romano, F.B., et al. A model for the generation and interconversion of ER morphologies. Proceedings of the National Academy of Sciences. 2014; 111(49):E5243-51.

47. Mukherjee, R., Chakrabarti, O. Ubiquitin-mediated regulation of the E3 ligase GP78 by MGRN1 in trans affects mitochondrial homeostasis. Journal Cell Science. 2016; 129(4):75773.

48. Nucifora, F.C., Sasaki, M., Peters, M.F., et al. Interference by huntingtin and atrophin-1 with cbp-mediated transcription leading to cellular toxicity. Science. 2001; 291(5512):24238.

49. Peters, C., Espinoza, M.P., Gallegos, S., Opazo, C., Aguayo, L.G. Alzheimer's A $\beta$ interacts with cellular prion protein inducing neuronal membrane damage and synaptotoxicity. Neurobiology of Aging. 2015; 36(3):1369-77.

50. Lindholm, D., Wootz, H., Korhonen, L. ER stress and neurodegenerative diseases. Cell Death and Differentiation. 2006; 13(3):385.

51. Joshi, G., Chi, Y., Huang, Z., Wang, Y. A $\beta$-induced Golgi fragmentation in Alzheimer's disease enhances A $\beta$ production. Proceedings of the National Academy of Sciences. 2014; 111(13):E1230-9.

52. Fifita, J.A., Williams, K.L., Sundaramoorthy, V., et al. A novel amyotrophic lateral sclerosis mutation in OPTN induces ER stress and Golgi fragmentation in vitro. Amyotrophic Lateral Sclerosis and Frontotemporal Degeneration. 2017; 18(1-2):126-33.

53. Jiang, Z., de Messieres, M., Lee, J.C. Membrane remodeling by $\alpha$-synuclein and effects on amyloid formation. Journal of the American Chemical Society. 2013; 135(43):15970-3. 
54. Pepe, A., Avolio, R., Matassa, D.S., et al. Regulation of sub-compartmental targeting and folding properties of the Prion-like protein Shadoo. Scientific Reports. 2017; 7(1):3731.

55. Kim, J.E., Li, S., GrandPré, T., Qiu, D., Strittmatter, S.M. Axon regeneration in young adult mice lacking Nogo-A/B. Neuron. 2003; 38(2):187-99.

56. Zhang, H., Hu, J. Shaping the endoplasmic reticulum into a social network. Trends in Cell Biology. 2016; 26(12):934-43.

57. Jozsef, L., Tashiro, K., Kuo, A., et al. Reticulon 4 is necessary for endoplasmic reticulum tubulation, STIM1-Orail coupling, and store-operated calcium entry. Journal of Biological Chemistry. 2014; 289(13):9380-95.

58. Rämö, O., Kumar, D., Gucciardo, E., et al. NOGO-A/RTN4A and NOGO-B/RTN4B are simultaneously expressed in epithelial, fibroblast and neuronal cells and maintain ER morphology. Scientific Reports. 2016; 6:35969.

59. Luis, L.P., Snapp, E.L., Denecke, J., Lippincott-Schwartz, J., Hawes, C., Brandizzi, F. Endoplasmic reticulum export sites and Golgi bodies behave as single mobile secretory units in plant cells. The Plant Cell. 2004; 16(7):1753-71.

60. Brandizzi, F., Snapp, E.L., Roberts, A.G., Lippincott-Schwartz, J., Hawes, C. Membrane protein transport between the endoplasmic reticulum and the Golgi in tobacco leaves is energy dependent but cytoskeleton independent: evidence from selective photobleaching. The Plant Cell. 2002; 14(6):1293-309.

61. Urra, H., Hetz, C. The ER in 4D: a novel stress pathway controlling endoplasmic reticulum membrane remodeling. Cell Death and Differentiation. 2012; 19(12):1893-5.

62. Varadarajan, S., Bampton, E.T., Smalley, J.L., et al. A novel cellular stress response characterised by a rapid reorganisation of membranes of the endoplasmic reticulum. Cell Death and Differentiation. 2012; 19(12):1896-907.

63. Hughes, H., Budnik, A., Schmidt, K., et al. Organisation of human ER-exit sites: requirements for the localisation of Sec16 to transitional ER. Journal of Cell Science. 2009; 122(16):2924-34. 
64. Peretti, D., Dahan, N., Shimoni, E., Hirschberg, K., Lev, S. Coordinated lipid transfer between the endoplasmic reticulum and the Golgi complex requires the VAP proteins and is essential for Golgi-mediated transport. Molecular Biology of the Cell. 2008; 19(9):3871-84.

65. Joensuu, M., Jokitalo, E. ER sheet-tubule balance is regulated by an array of actin filaments and microtubules. Experimental Cell Research. 2015; 337(2):170.

66. Chen, S., Cui, Y., Parashar, S., Novick, P.J., Ferro-Novick, S. ER-phagy requires Lnp1, a protein that stabilizes rearrangements of the ER network. Proceedings of the National Academy of Sciences. 2018; 115(27):E6237-44.

67. Wang, S., Tukachinsky, H., Romano, F.B., Rapoport, T.A. Cooperation of the ERshaping proteins atlastin, lunapark, and reticulons to generate a tubular membrane network. Elife. 2016; 5:e18605.

68. Srivastava, D., Chakrabarti, O. Mahogunin-mediated $\alpha$-tubulin ubiquitination via noncanonical K6 linkage regulates microtubule stability and mitotic spindle orientation. Cell Death \& Disease. 2014; 5(2):e1064.

69. Mukherjee, R., Majumder, P., Chakrabarti, O. MGRN1-mediated ubiquitination of $\alpha$-tubulin regulates microtubule dynamics and intracellular transport. Traffic. 2017; 18(12):791-807.

70. Forster, J.I., Köglsberger, S., Trefois, C., et al. Characterization of differentiated SHSY5Y as neuronal screening model reveals increased oxidative vulnerability. Journal of Biomolecular Screening. 2016; 21(5):496-509.

71. English, A.R., Voeltz, G.K. Rab10 GTPase regulates ER dynamics and morphology. Nature Cell Biology. 2013; 15(2):169.

72. Gonzalez, R.C., Woods, R.E., Eddins, S.L. (2003). Digital Image Processing Using MATLAB. Prentice-Hall, Inc., Upper Saddle River, NJ, USA.

73. Hailey, D.W., Rambold, A.S., Satpute-Krishnan, P., et al. Mitochondria supply membranes for autophagosome biogenesis during starvation. Cell. 2010; 141(4):656-67.

74. Apweiler, R., Bairoch, A., Wu, C.H., et al. UniProt: the universal protein knowledgebase. Nucleic Acids Research. 2004; 32(suppl_1):D115-9. 


\section{Figure Legends}

Figure 1: Presence of cytosolic PrP aggregates and $\operatorname{PrP}$ variants with weak signal sequences affect ER morphology and dynamics.

(A) HeLa cells cotransfected with ER-GFP, RFP tagged cyPrP, and PrP variants with different signal sequences (Prl-PrP, wt-PrP, Ifn $\gamma-\operatorname{PrP}$ ) were imaged $48 \mathrm{~h}$ after transfection. White arrows mark ER vacuolation. Note fragmentation and vacuolation of the ER network in cells expressing wt-PrP, and Ifn $\gamma$-PrP. Cells transfected with only cyPrP shows an intact ER with little or no fragmentation. Percentage of cells with vacuolated ER was plotted. Data represents $\sim 80$ cells per set from 3 independent experiments. Error bars, standard error. Scale bar $5 \mu \mathrm{m}$.

(B) Live cell imaging of HeLa cells cotransfected with ER-GFP, RFP tagged cyPrP, and PrP variants of different signal sequences (Prl-PrP, wt-PrP, Ifn $\gamma$-PrP).White arrows mark the sites of fusion events. Expression of RFP tagged cyPrP and ER-GFP is shown in lower panel. The number of tubular ER extension events that occurred in $10 \mu \mathrm{m}^{2}$ ROIs over a $5 \mathrm{~min}$ time course were scored as either resulting in a successful or unsuccessful fusion event. The percentage of successful fusion events over total number of events was plotted. Data is representative of 100 ROIs measured from $\sim 30$ cells per set, taken from 3 independent experiments. Error bars, standard error. Scale bar $5 \mu \mathrm{m}$.

(C) HeLa cells cotransfected with ER-GFP, RFP tagged cyPrP, and indicated PrP variants were subjected to FRAP assay $48 \mathrm{~h}$ after transfection. $5 \mu \mathrm{m}^{2}$ ROIs (marked by white boxes) were marked followed by photobleaching and time lapse imaging to monitor fluorescent recovery. Expression of RFP tagged cyPrP and ER-GFP is shown in lower panel. Scale bar $5 \mu \mathrm{m}$.

(D) Percent recovery after photobleaching was plotted from images in C. Data represents 20 cells per set from 3 independent experiments. Error bars indicate standard deviation. *** $\mathrm{p} \leq 0.001, * \mathrm{p} \leq 0.05$, using unpaired 2-tailed Student's t-test. All $\mathrm{p}$ values are with respect to wt-PrP. 
Figure 2: Actin remodelling in cells with cytosolic aggregates and high load of nontranslocated proteins.

(A) HeLa cells transfected with PrP variants and expressing aggregates of PrP or Htt (CFP tagged cyPrP/ $\left.\mathrm{Htt}_{\mathrm{Q} 103}-\mathrm{GFP}\right)$ were fixed $48 \mathrm{~h}$ post-transfetion, stained with Alexa568 tagged Phalloidin, visualised by confocal imaging and depicted as 3-D projections. Long actin filaments are seen in Prl-PrP expressing cells. Presence of wt-PrP and Ifn $\gamma$-PrP results in a more punctuate actin staining. The zoomed in images represent these phenotypes. For ease of visualization the actin filaments are demonstrated in gray. Data represents $\sim 85$ cells per set from 3 independent experiments. Scale bar $5 \mu \mathrm{m}$.

(B) The graph analysed with data from panel (A) indicates percentage of cells with long filamentous actin as compared to those with punctuate actin..

(C) Analyses of the actin-polymerization state through centrifugation mediated separation of F- and G-actin under the indicated conditions. Data represents mean of 3 independent experiments. ns, not significant, ${ }^{* * *} \mathrm{p} \leq 0.001$ using Student's t-test. Error bars, \pm SEM.

Figure 3: Fragmentation of Golgi in cells with cytosolic protein aggregates and PrP variants.

(A) HeLa cells cotransfected with GFP tagged cyPrP, and indicated PrP variants were fixed $48 \mathrm{~h}$ after transfection and immunostained with TRAP $\alpha$ and GM130 antibodies. Note fragmentation of Golgi in cells expressing wt-PrP, and Ifn $\gamma$-PrP as reflected in the snapshots of z-stack images. For ease of visualization the aggregates are represented in gray, TRAP $\alpha$ in green, GM130 in red and DAPI in blue. Data represents $\sim 100$ cells per set from 3 independent experiments. Scale bar $5 \mu \mathrm{m}$.

(B) HeLa cells cotransfected with $\mathrm{Htt}_{\mathrm{Q} 103}-\mathrm{GFP}$ and $\operatorname{PrP}$ variants were fixed $48 \mathrm{~h}$ after transfection and immunostained similar to (A); snapshots of $\mathrm{z}$-stacks images from are represented. For ease of visualization the aggregates has been represented in gray, TRAP $\alpha$ in green, RTN4 in red and DAPI in blue. Data represents $\sim 85$ cells per set from 3 independent experiments. Scale bar $5 \mu \mathrm{m}$.

(C) Histogram plots data from panels (A) and (B). Error bars, \pm SEM. 


\section{Figure 4: RTN4 colocalises with cyPrP aggregates in wt-PrP and Ifn $\gamma$-PrP expressing}

cells.

(A) HeLa cells cotransfected with RFP tagged cyPrP, and indicated PrP variants were grown in culture for $48 \mathrm{~h}$. They were then subjected to digitonin fractionation to separate cytosolic, membrane and detergent insoluble fractions. These fractions were then immunoblotted with RTN4 and RFP antibodies. Note higher proportion of RTN4 in aggregates in wt-PrP and Ifn $\gamma$-PrP expressing cells.

(B) Immunoblot in A was quantitated to plot the proportion of RTN4 in detergent insoluble fractions. Data represents mean of 3 independent experiments. Error bars, standard deviation.

(C) Cells transfected similarly as in (A) were permeabilised with digitonin, both digitonin permeabilised and intact cells were fixed with $4 \%$ formaldehyde and immunostained with RTN4 antibody. Aggregates are marked in red and RTN4 staining is marked in green. The insets indicate enhanced retention of RTN4 on cyPrP aggregates in cells with wt-PrP and Ifn $\gamma$-PrP. Scale bar $5 \mu \mathrm{m}$.

(D) Percentage of RTN4 positive aggregates was plotted from images in (C). 75 aggregates were analysed from $\sim 35$ cells taken from 3 independent experiments. Error bars, standard deviation.

(E) A similar experiment was performed as in (C) with CRFR1 or Prl-CRFR1, instead of PrP variants; cells were analysed $96 \mathrm{~h}$ post-transfection. Insets are shown to better visualise the aggregates. Scale bar $5 \mu \mathrm{m}$.

(F) Histogram depicts percentage of RTN4 positive aggregates from images in (E). 60 aggregates were analysed from $\sim 30$ cells taken from 3 independent experiments. Error bars, standard deviation.

\section{Figure 5: Presence of nontranslocated proteins perturb three-way ER junctions}

HeLa cells cotransfected with GFP tagged cyPrP, KDEL-mRuby and PrP variants were grown in culture for $48 \mathrm{~h}$. They were then imaged in live for 5 minutes. The zoomed in images highlight the three-way junctions. Note disruption of the three-way junctions in cells expressing wt-PrP, and Ifn $\gamma-\operatorname{PrP}$. Cells transfected with only Prl-PrP show presence of stable 
three-way junctions with little or no fragmentation. For ease of visualization the aggregates are represented in gray. Scale bar $5 \mu \mathrm{m}$.

The number of stable three way junctions per $100 \mathrm{um}^{2}$ was plotted. Data represents $\sim 90$ cells per set from 4 independent experiments. Error bars indicate standard deviation.

\section{Figure 6: Presence of nontranslocated proteins differentially affects the three-way junctions at the RER and SER}

(A) HeLa cells cotransfected with RFP tagged cyPrP, RTN4-GFP and indicated PrP variants were grown in culture for $48 \mathrm{~h}$. They were then fixed and stained against LNPK. Note number of LNPK positive puncta on RTN4 is maximum in presence of Prl-PrP while least for Ifn $\gamma$ PrP. For ease of visualization the aggregates are indicated in gray, RTN4-GFP in red and LNPK in green. Scale bar $5 \mu \mathrm{m}$.

(B) HeLa cells cotransfected with RFP tagged cyPrP, Sec61- $\beta$-GFP and PrP variants were grown, fixed and stained against LNPK as before. Note number of LNPK positive puncta on Sec61- $\beta$-GFP was the most for Ifn $\gamma$-PrP. For ease of visualization the aggregates are indicated in gray, Sec61- $\beta$-GFP in red and LNPK in green. Scale bar $5 \mu \mathrm{m}$.

(C) The result of (A and B) have been quantitated here with data of $\sim 50$ cells per set from 3 independent experiments. Error bars indicate standard deviation.

(D) The graph indicates the percentage of cells with LNPK on sheets versus tubules. Error bars indicate standard deviation.

\section{Figure 7: Schematic diagram summarizing the results}

Nontranslocated protein precursors in the presence cytosolic protein aggregates perturb endoplasmic reticulum morphology and dynamics. The propensity of RTN proteins for aggregation is enhanced in the presence of nontranslocated proteins. This disrupts three-way junctions and leads to fragmentation of the ER. 
Figure 1

(A)
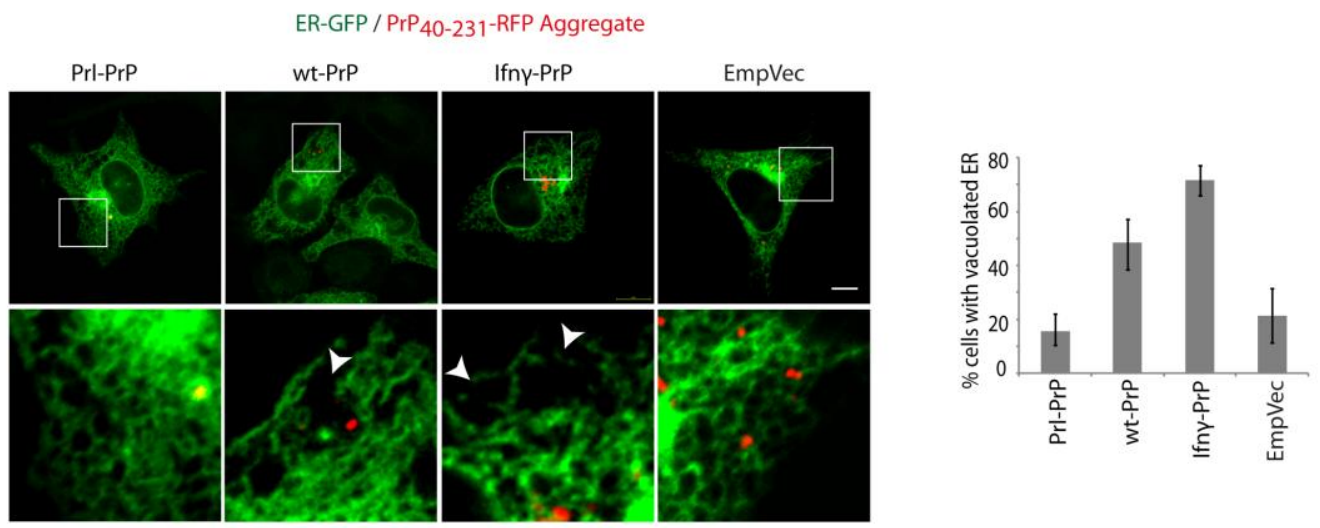

(B)

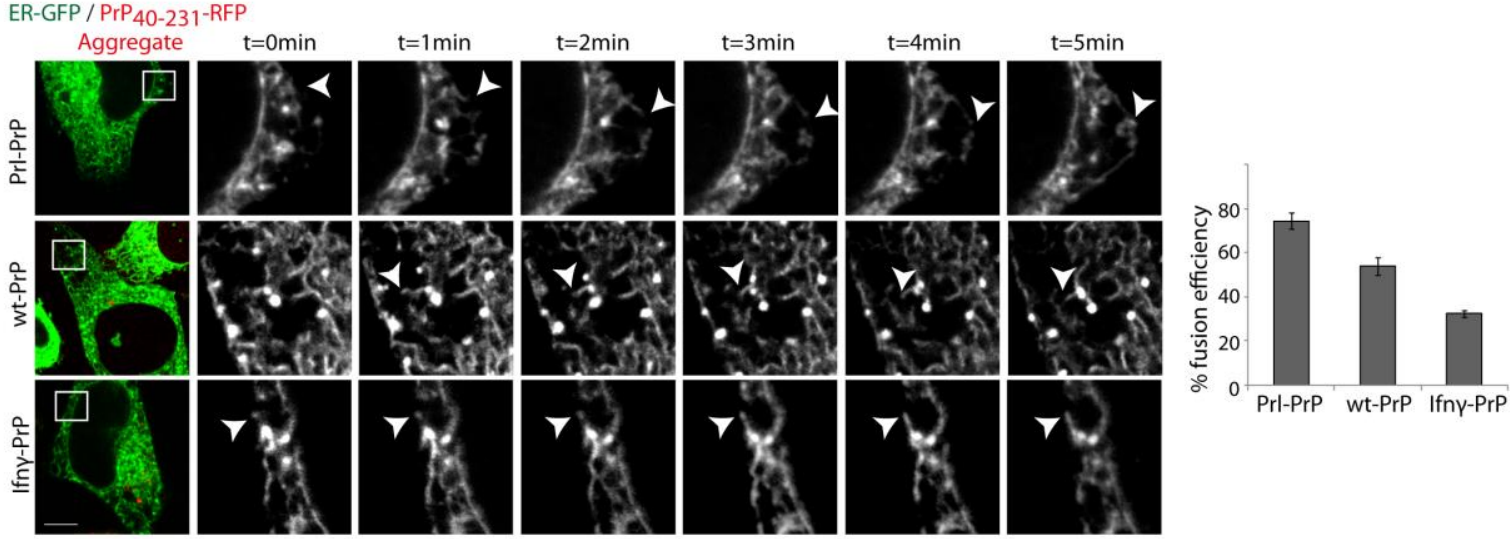

(C)

(D)
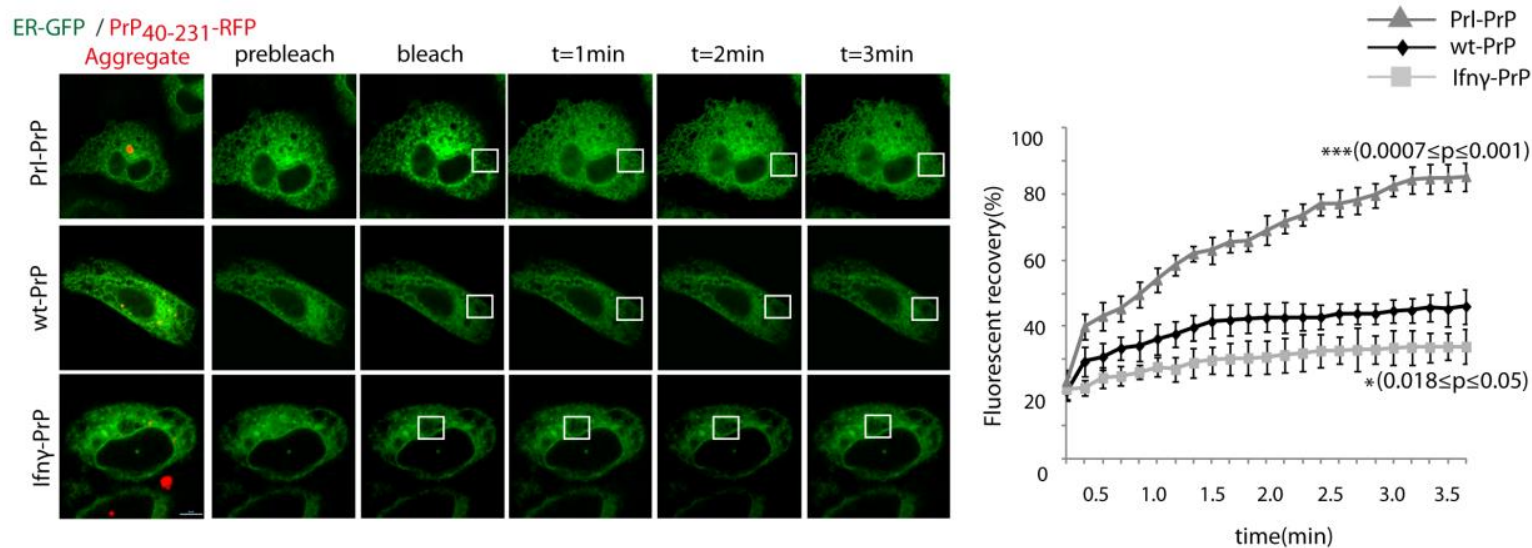
Figure 2

(A)
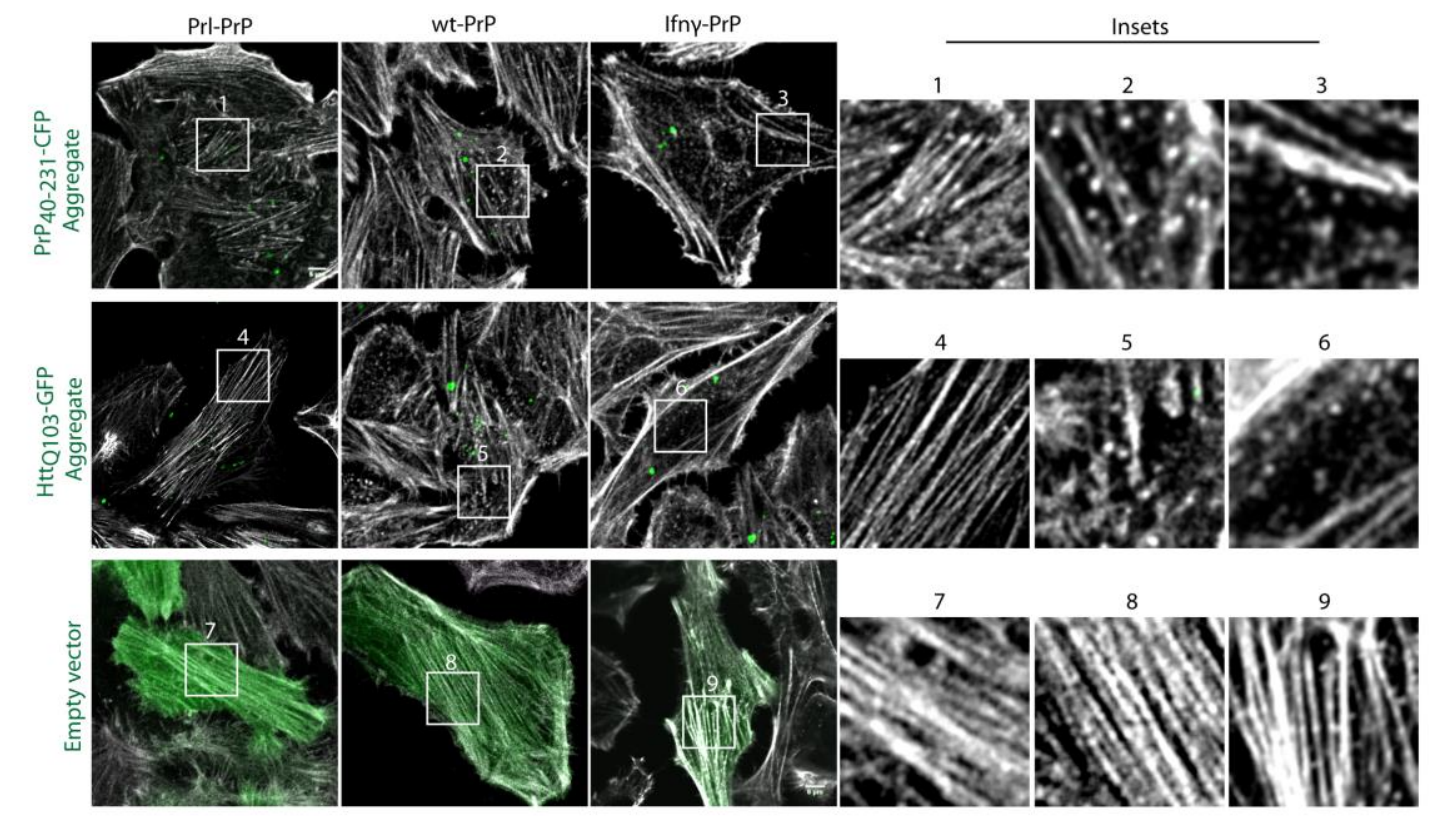

(B)

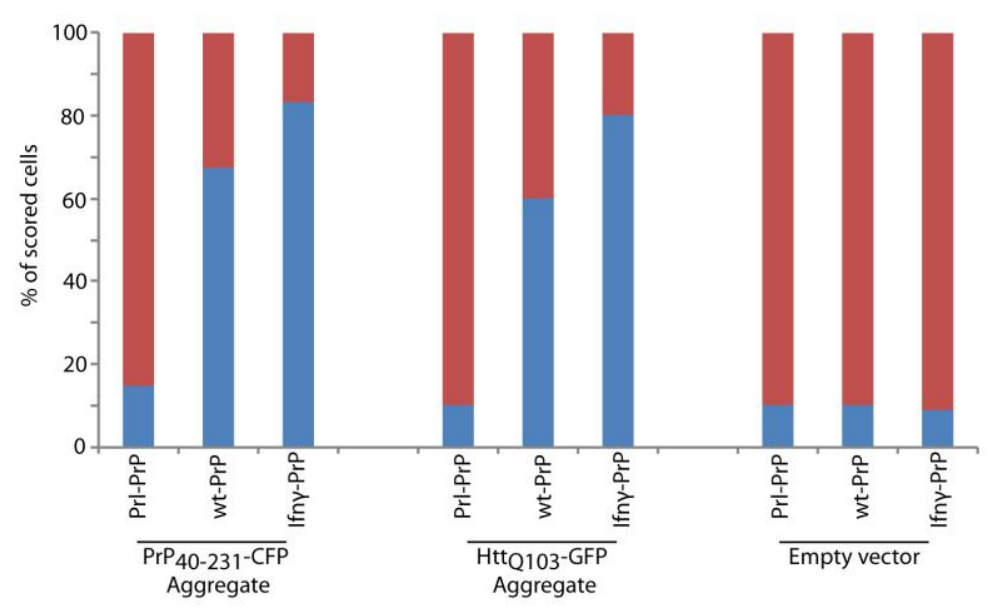

Long actin filaments

Punctuate actin

(C)
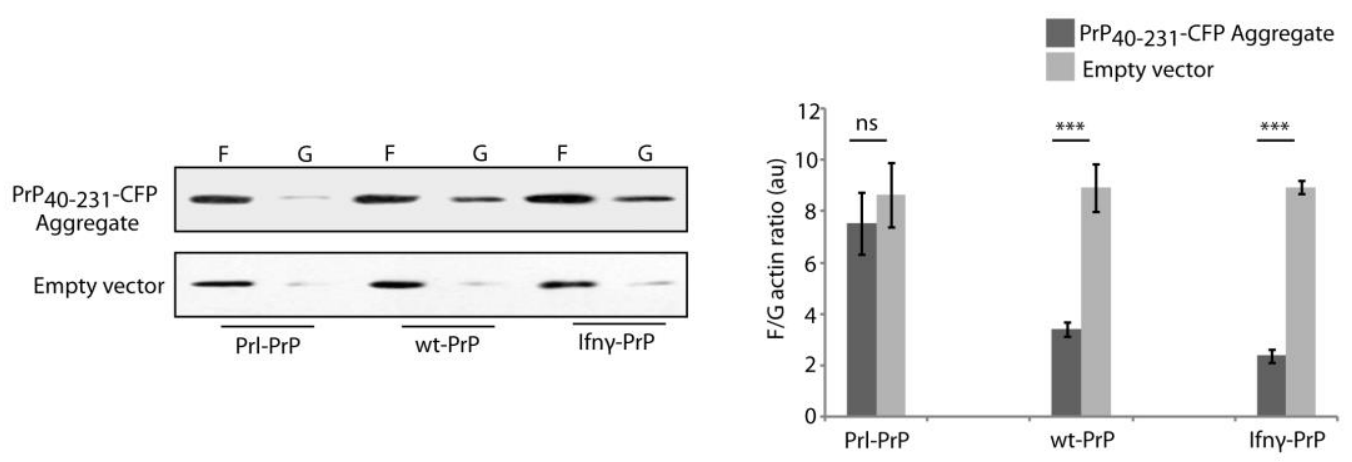
Figure 3

(A)

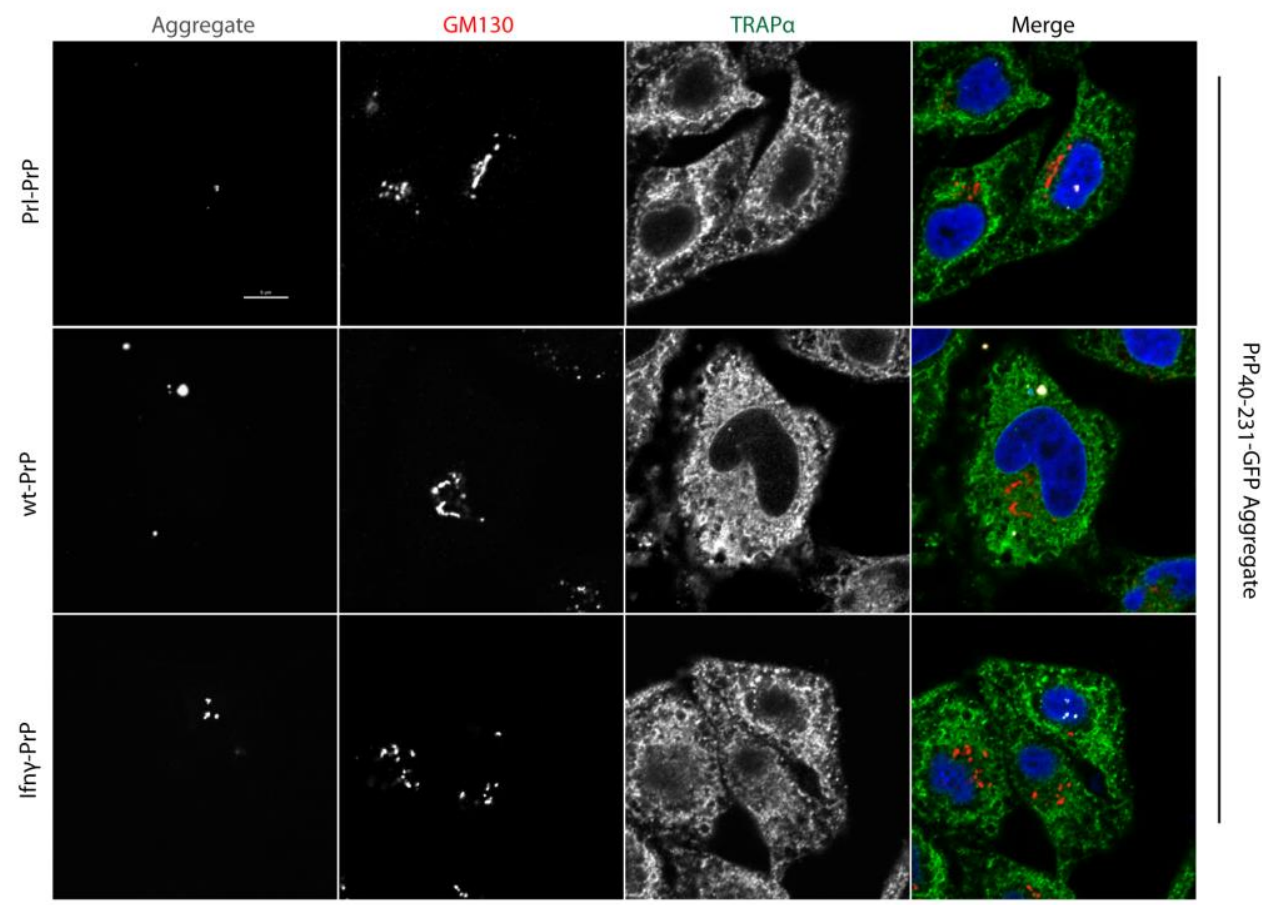

(B)

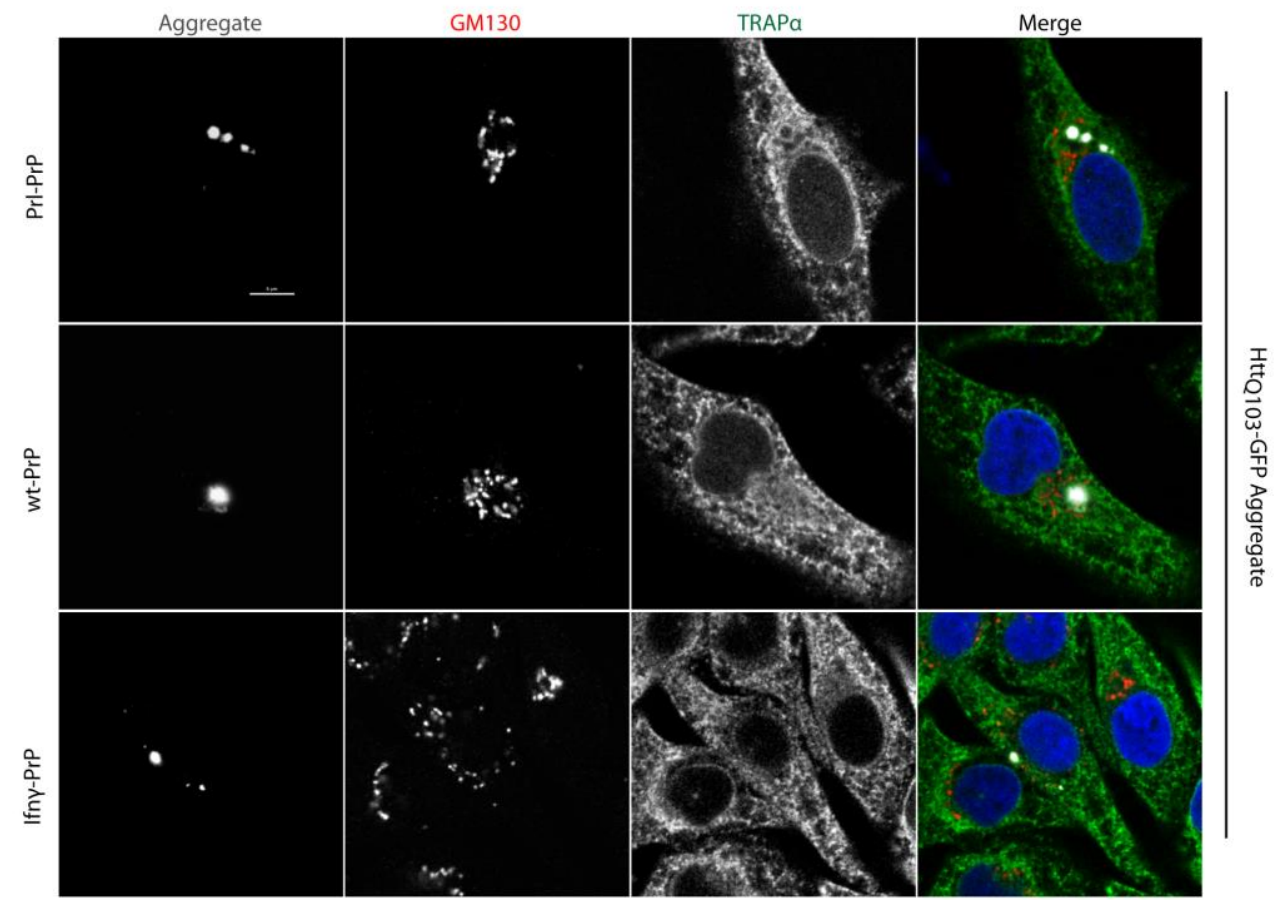

(C)

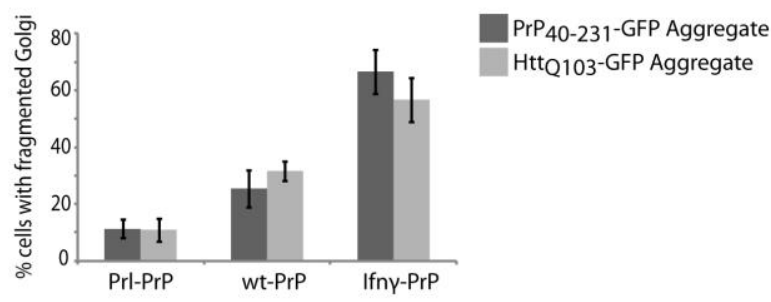


Figure 5

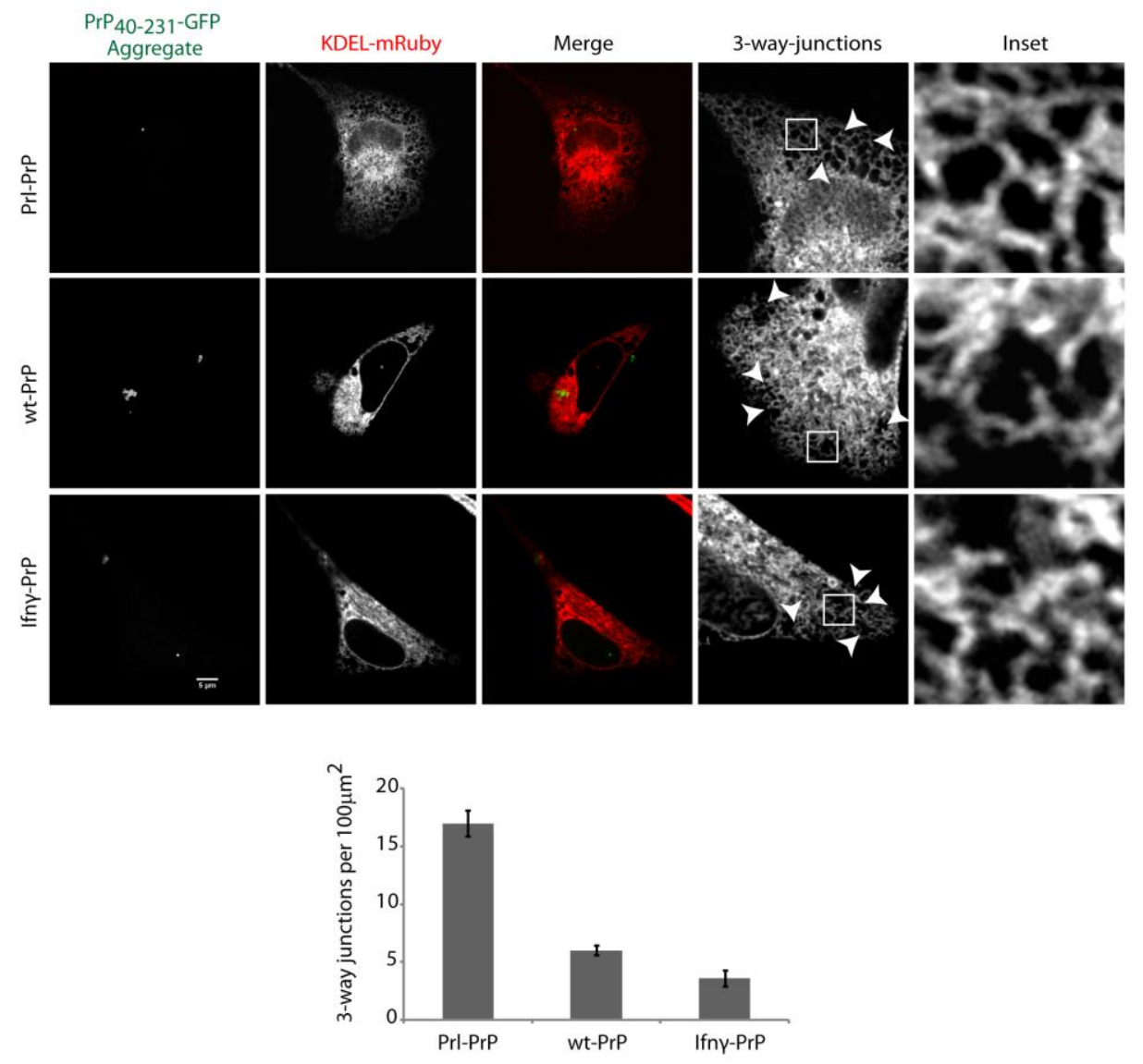


Figure 6
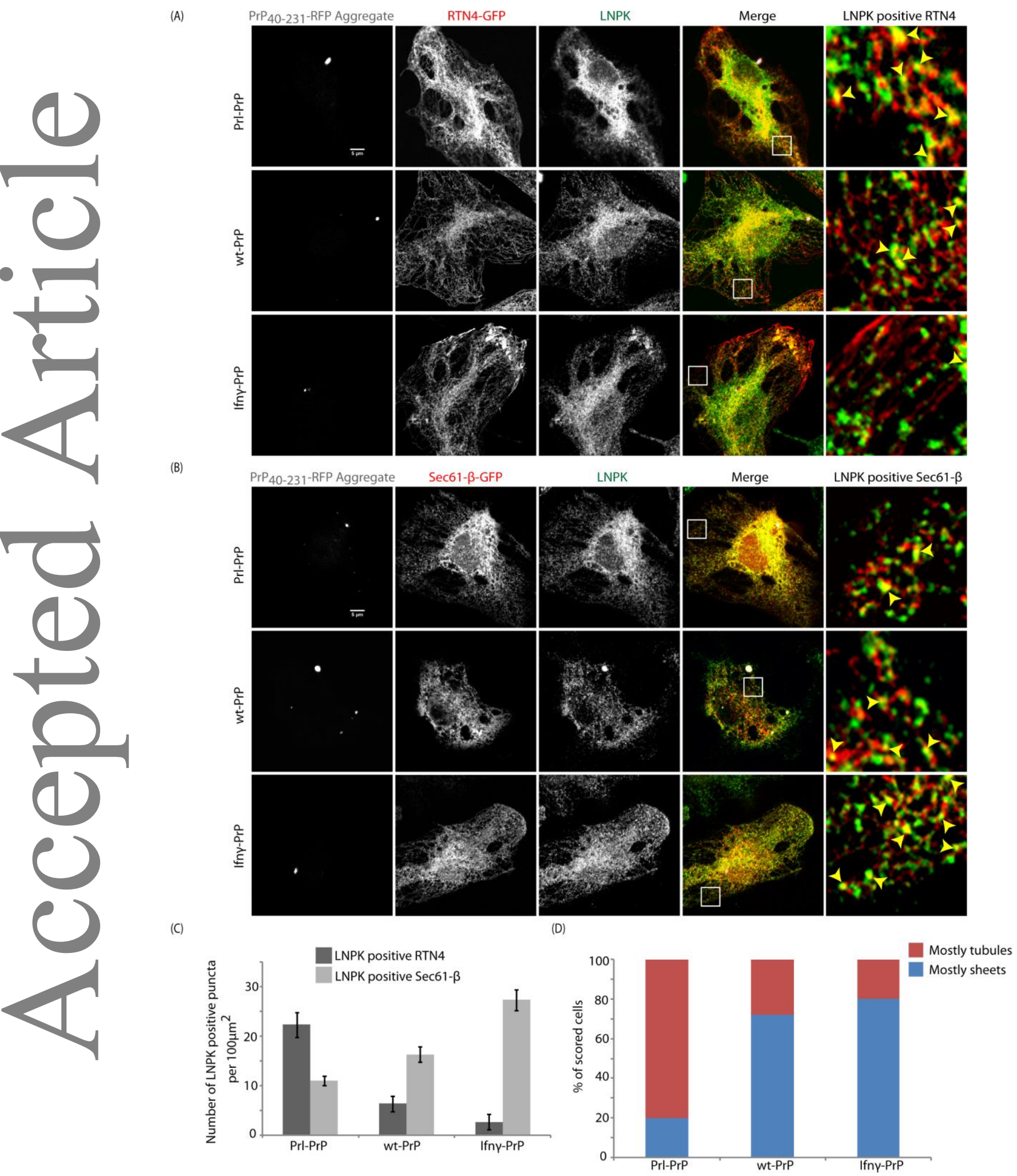